\title{
A Novel Pilot Spoofing Scheme via Intelligent Reflecting Surface Based On Statistical CSI
}

\author{
Jie Yang, Xinsheng Ji, Kaizhi Huang, Xiaoli Sun Xiaoming Xu
}

\begin{abstract}
Pilot spoofing attack brings challenges to the physical layer secure transmission. However, since the inherent characteristics of wireless environment have not changed, active eavesdropping can be detected based on prior information. Intelligent reflecting surface (IRS), with the real-time programmable characteristics for wireless environment, provides new possibilities for effective pilot spoofing. In this paper, the IRS is deployed near the legitimate users and the control strategy is embeded into the legitimate communication process under time-division duplex (TDD) mode to assist eavesdroppers to implement pilot spoofing. By designing different phase shifts at the IRS during the uplink phase and downlink phase, the channel reciprocity between uplink and downlink disappears, and thus the secure beamforming vector is biased towards the eavesdropper. Furthermore, in order to obtain more information, the average secrecy rate based on the statistical channel state information is established by carefully designing the phase shifts. The formulated problem is non-trivial to solve. By using alternating optimization and Charnes-Cooper transformation technique, the original problem is transformed into convex form and a sub-optimal solution is achieved. Finally, simulation results show that our proposed scheme poses serious secure threat for TDD systems.
\end{abstract}

Index Terms-Pilot spoofing, Intelligent reflecting surface, Average secrecy rate, Charnes-Cooper transformation, Alternating optimization

\section{INTRODUCTION}

$\mathbf{R}$ ECENTLY, the openness of wireless communication provides high-speed data transmission for our daily lifes, while it also brings the risk of information being eavesdropped. As a complement to the conventional cryptographic techniques, physical layer security (PLS) technology, which exploits the channel differences between different users to realize secure communication, has attracted growing attention [1]. Specifically, secure beamforming (SB) technique, which exploits multiple antennas to enhance the communication signal at the legitimate user and degrade the signal quality at the eavesdropper, is a well-known approach. Moreover, with the application of 5G, Massive Multiple-Input MultipleOutput technology is proposed, which can provide more spatial degrees of freedom to improve secrecy capacity.

However, there exist some shortcomings for this technology, which may be utilized by eavesdroppers. As we know, accurate channel state information (CSI) is an essential prerequisite in

This work was supported in part by the National Natural Science Foundation of China under Grant 61801435 and Grant 61701538, and in part by China Postdoctoral Science Foundation 2019M663994.(Corresponding author: Kaizhi Huang);

J. Yang, X. Ji, K. Huang, X. Sun, X Xu are with the School of Information Engineering University, Zhengzhou, 450002, China.(e-mails: yj_csu@126.com, jxs@ndsc.com.cn, huangkaizhi@tsinghua.org.cn, lgdxsunxiaoli@sina.com, ee_xiaomingxu@sina.com). designing SB vectors. Different from passive eavesdropper, which only wiretap information, active eavesdropper can attack the channel training phase of legitimate links to improve wiretapping performance, which is a serious threat especially for time-division duplex (TDD) systems. In a TDD system, the process of obtaining downlink CSI is breifly descripted as follows: the legitimate receiver (LR) transmits pilot symbols to the legitimate transmitter (LT) during uplink training phase. Then, in order to reduce the interaction overhead, the estimated uplink channel is regarded as the downlink channel by exploiting reciprocity at a coherent time, and SB vector based on this CSI is designed and utilized to transmit the confidential message to the LR. If an eavesdropper attacks the uplink training phase by transmitting the same pre-designed training sequence as the LR, the estimated channel obtained at the LT is a weighted combination of the legitimate channel and the wiretap channel. Based on this faked CSI, the beam formed by the BS will be oriented towards both the LR and the Eve, which results in severe signal leakage to Eve. In [2], the authors first proposes this active eavesdropping method and name it as pilot spoofing attack (PSA). Then its damages are analyzed. In [3], a PSA approach carried out by multiple eavesdroppers is investigated in a TDD system. And during the uplink channel training phase, multiple Eves collaboratively impair the channel acquisition of the legitimate link, aiming at maximizing the wiretapping signal-to-noise ratio (SNR) in the subsequent downlink data transmission phase. Two different scenarios are investigated: one is that the BS was unaware of the PSA, and the other is that the BS attempted to detect the presence of the PSA. Moreover, the PSA detection scheme is also investigated. In [4], the authors formulated the PSA detection as a binary hypothesis testing problem, and the likelihood ratio based on the energy of the received signal is used as the detection statistic. In [5], under the same pilot allocation protocol, the authors respectively proposes a random channel training scheme and a jamming-resistant scheme employing an unused pilot sequence to combat the pilot contamination attack and to maintain secure communication. The reasons that these methods work rely on a key assumption: the received combination pilot signal contains channel characteristics of the eavesdropper, which LT can use some method to distinguish. Further, from the internal reasons, the conventional pilot spoofing attack does not really change the reciprocity of the uplink and downlink channels. To fundamentally avoid being detected by legitimate users, a novel attack method which can reconstruct the wireless communication environment in real time and change the reciprocity characteristics should be considered. 
Meanwhile, Intelligent reflective surface (IRS), which can reconstruct wireless propagation environment in real time, has been viewed as a key emerging technology for $6 \mathrm{G}$ wireless networks [6]. IRS is a planar array consisting of a large number of low-cost passive elements and each element can adjust the reflection phase shift on the incident signal independently whereby the reflected signal can be enhanced or weakened at given users. Besides, the switching time of the state at each unit can be low to the order of microsecond $(\mu \mathrm{s})$ [7], which is much smaller than the typical channel coherence time that is on the order of millisecond (ms), and thus IRS is well suited for mobile applications for time-varying channels. Therefore, as a novel promising solution, IRS has been investigated in various applications such as coverage extension [8], physical layer security [9] [10], energy efficiency improvement [11], and so on. However, few literatures consider reverse application, where IRS is used for enhancing eavesdropping. In [12], the IRS is utilized as a jammer to sabotage the legitimate communication system without any energy footprint. While, these works just simply consider deploying the IRS around the LT or the LR, and tune the phase shifts according to the instantaneous CSI.

Actually, due to the openness of the communication protocol, the control strategy of the IRS is embedded in the protocol and change the reciprocity of the uplink and downlink channels, which may bring new threat to legal communication. Moreover, with large low-cost passive reflecting elements, more design parameters brought by IRS can be utilized to moderate link quality and the problem is usually intractable. To the best of our knowledge, by far there is few work that considers IRS in pilot spoofing scenario. Motived by this, a novel adverse application of IRS is investigated for pilot spoofing. The control protocol of the IRS is embeded into the legitimate protocol. Consequently, this novel active attack can lead to information leakage without leaving any energy footprint, and thus it is very difficult to detect and prevent such kind of attacks. Our main contributions are summarized as follows:

1) A novel pilot attack scheme in a three-node model with the aid of the IRS is proposed. In this model, the IRS is covertly deployed the LT and remotely controlled by the eavesdropper. During uplink phase and downlink phase, the phase shifts at the IRS are different, and thus the downlink CSI estimated by the LT is different from the actual downlink CSI, the designed SB vector will be shifted from LR. Furthermore, by carefully predesigning the phase shifts, more information can be leaked to the eavesdropper.

2) The average secrecy rate minimization problem based on statistical CSI is formulated and solved. Since instantaneous information is difficult to obtain for eavesdroppers, the average secrecy rate is considered as the performance metric and the optimization problem is established by jointly designing phase shifts at the uplink phase and downlink phase. First, the approximate expression of the problem is derived, then non-convex problem is efficiently solved by exploiting alternating optimization algorithm and Charnes-Cooper transformation method. Moreover, a sub-optimal iterative algorithm is proposed and the computational complexity is analyzed.

3) Numerical results show the impact of parameters, such as the transmit power, the Rician factor, on system performance. Compared with the existing pilot attack schemes, our proposed scheme can significantly enhance the performance of eavesdroppers. As the number of transmit antennas increases, IRS brings more serious threat for secrecy. Moreover, when the number of low cost reflecting units increases, the secrecy performance can be further cut down, which further illustrates the advantages of the solution.

The remainder of this paper is organized as follows. Section II introduces the system model and proposes the pilot spoofing attack scheme via IRS. Section III establishes the optimization problem model and solves the optimization problem. Section IV provides the simulation results. Section V analyzes the advantages of our scheme and explores possible detection and countermeasure methods. Section VI provides conclusions.

\section{SYSTEM MODEL AND SCHEME ANALYSIS}

\section{A. System Model}

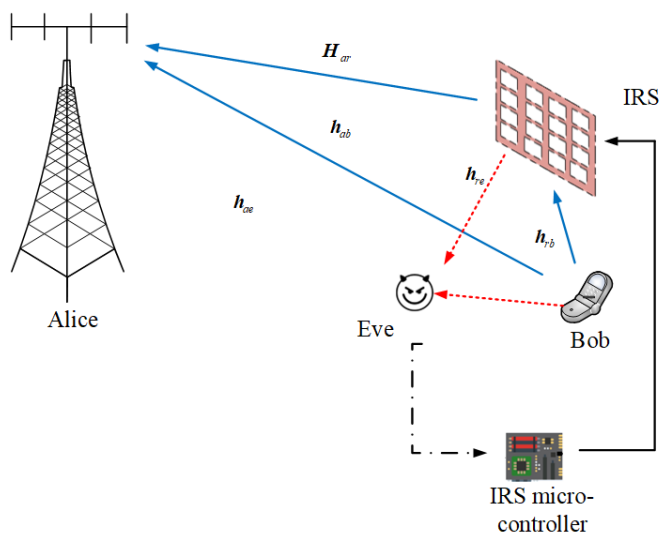

(a) uplink phase

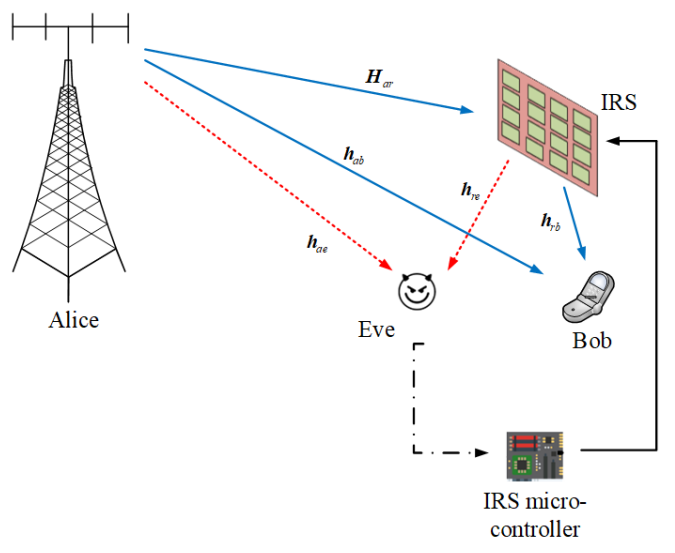

(b) downlink phase

Fig. 1: IRS-assisted Pilot Spoofing Attack Model 
As depicted in Fig.1, we consider a three-node pointto-point communication model, where the transmitter Alice communicates with the receiver Bob under the TDD mode, and the eavesdropper Eve tries to overhear the signal. Alice is equipped with $M_{a}$ antennas, where $M_{a}>1$, while Bob and Eve is equipped with single antenna, respectively. Meanwhile, IRS is deployed near Alice and is controlled remotely by Eve through a private wireless channel. The IRS is composed of $N$ reflecting units and $\Phi=\operatorname{diag}\left(\beta_{1} e^{j \theta_{1}}, \beta_{2} e^{j \theta_{2}}, \cdots \beta_{N} e^{j \theta_{N}}\right)$ is denoted as the reflection coefficient matrix at the IRS, where assuming that the amplitude is always 1 , namely $\beta_{i}=1$, $\theta_{n} \in[0,2 \pi]$ denotes the phase shift on the incident signal at its $n$-th element. Suppose that there exist some low-power sensors integrated into the IRS and the reflected channel state information can be probed by these detectors [13] [14]. Different from [2], where Eve sends jamming signal during the uplink pilot phase to induce Alice to obtain the false uplink CSI, in this model, Eve only remotely controls the IRS to change its phase shifts several times in a coherent time to conduct pilot spoofing attack. The specific implementation process is shown in Fig.2:

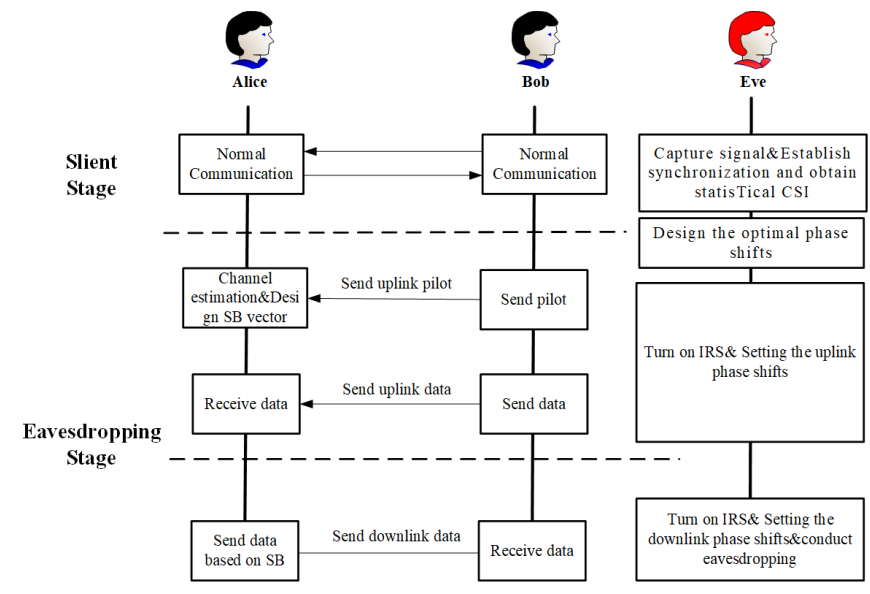

Fig. 2: Flow chart of pilot spoofing attack via IRS

\section{1)Silent stage:}

Alice and Bob communicate normally, while Eve keeps silent and only tries to establish synchronization with legitimate users. Although the received signal by Eve is too weak to decode information, the timing synchronization is easy to be found by Eve due to the open communication protocol after a long time capture.

\section{2)Eavesdropping stage:}

(1)Before Bob begins to send the uplink pilot, Eve turns on the IRS and sets the uplink phase shifts at the IRS. Then during the uplink pilot slot, Bob sends the pilot to Alice, and then Alice estimates the uplink CSI after receiving the pilot. In this case, the estimated CSI includes the direct path and the reflected path from Alice to Bob, as shown in Fig 1(a). Then due to the channel reciprocity between the uplink and the downlink, Alice utilizes the uplink probed CSI as the downlink CSI, and design the SB vector.

(2) In the uplink data slot, Bob sends uplink data to Alice. Alice performs channel equalization and information decoding.
At this time, the phase shifts are maintained.

(3)Before Alice starts to send downlink data, Eve sets the downlink phase shifts at the IRS. In the downlink phase, Alice performs secure beamforming to send confidential signal, as shown in Fig 1(b), while Eve tries to overhearing data.

As can be seen from the above process, if we set different phase shifts at the uplink phase and downlink phase, the channel reciprocity disappears. Then the uplink CSI probed by Alice is no longer consistent with the actual downlink CSI. Since the SB vector is designed based on the uplink CSI, the downlink beam cannot be aligned with Bob, resulting in information leakage. At the same time, by carefully designing the phase shifts at different stage, the beam can be adjusted to Eve, and more information is leaked to Eve, thus degrading the security performance.

\section{B. The analysis of the Scheme}

The physical layer secure transmission technology constructs endogenous security based on location-based wireless environment differences, while the proposed scheme utilizes the agility characteristics of the IRS to reconstruct the wireless environment to combat physical layer security technique. Compared with the existing pilot spoofing attack methods, our scheme has the following advantages:

1) It is easy to imagine trying to find a similar way to replace the IRS to reconstruct the wireless environment, for example, deploying controllable mirrors or moving small objects to perform different actions during the uplink or downlink slot. These methods seems theoretically feasible. However, compared with IRS, there exist two problems. Firstly, precise timing control is difficult to realize, especially for high speed transmission. To achieve synchronization, a complex mechanical control structure is required, while for the IRS only a micro-controller is needed. Secondly, the IRS can change the phase of the incident signal and by designing the optimal reflection coefficients, the difference between uplink and downlink channel can be maximized to satisfy eavesdropping requirement. However, the channels reconstructed by other objects are uncontrollable. There is no guarantee that the transmit beam is deflected towards the eavesdropper, and it may even lead to receiving worse signal for the eavesdropper.

2) For traditional pilot attack methods, the pilot sent by the eavesdropper contains statistical CSI based on its location, and some detection methods can detect such an attack based on these prior information. For our proposed scheme, since the control strategy of the IRS is seamlessly embedded in the legitimate communication process, legitimate users working in TDD mode cannot perceive the difference between uplink channel and downlink channel, and the received pilot signal contain nothing about eavesdroppers locatoin information. Then the existing detection method is invalid.

3) Existing pilot attack methods require additional energy consumption when sending spoofing pilot, which is not conducive to the concealment of eavesdropping. While, due to the passive nature of the IRS, our solution does not require additional energy consumption except for its own state switching, which further increases the difficulty of detection. 
Therefore, our scheme provides new possibilities for implementing more effective pilot spoofing. Moreover, it is worth studying to eavesdropping capability maximizion under this scheme, which will be discussed in the following section.

\section{Problem Formulation ANd Solved}

\section{A. Problem Formulation}

In this section, We consider exploiting the IRS to construct the difference between the uplink and downlink channels which is beneficial to the eavesdropper. The three node signal model with IRS is firstly analyzed. All the channels are respectively expressed as follows: the channel from Alice to Bob is consist of a direct channel and a reflection channel, where the direct channel is denoted by $\mathbf{h}_{a b}^{H} \in{ }^{1 \times M_{a}}$, and the reflection channel is consist of two parts, the channel from Alice to IRS is denoted by $\mathbf{H}_{a r}^{H} \in N \times M$, the channel from IRS to Bob is denoted by $\mathbf{h}_{r b}^{H} \in{ }^{1 \times N}$. The channel from Alice to Eve is also consist of the reflection channel and the direct channel, where the direct channel is denoted by $\mathbf{h}_{a e}^{H} \in 1 \times M_{a}$, and the reflection channel is consist of two parts, the channel from Alice to IRS $\mathbf{H}_{a r}^{H}$, the channel from IRS to Eve is denoted by $\mathbf{h}_{r e}^{H} \in{ }^{1 \times N}$.

In the uplink phase, Eve turns on the IRS and sets the phase shifts as $\Phi_{1}$, then the uplink CSI estimated by Alice is expressed as

$$
\mathbf{h}_{a b u}^{H}=\mathbf{h}_{a b}^{H}+\mathbf{h}_{r b}^{H} \Phi_{1} \mathbf{H}_{a r}
$$

With the probed CSI $\mathbf{h}_{a b u}^{H}$, the downlink transmit SB vector is designed based on the MRT criterion and is given by

$$
\mathbf{w}=\sqrt{P_{t}} \frac{\mathbf{h}_{a b u}}{\left\|\mathbf{h}_{a b u}\right\|}=\sqrt{P_{t}} \frac{\left(\mathbf{h}_{a b}^{H}+\mathbf{h}_{r b}^{H} \Phi_{1} \mathbf{H}_{a r}\right)^{H}}{\left\|\mathbf{h}_{a b}^{H}+\mathbf{h}_{r b}^{H} \Phi_{1} \mathbf{H}_{a r}\right\|}
$$

where $P_{t}$ denotes the transmit power by Alice.

In the downlink phase, Eve sets the phase shifts as $\Phi_{2}$, then the received signal at Bob is expressed as:

$$
y_{b}=\left(\mathbf{h}_{a b}^{H}+\mathbf{h}_{r b}^{H} \Phi_{2} \mathbf{H}_{a r}\right) \mathbf{w} s+n_{b}
$$

where $n_{b} \sim \mathcal{C N}\left(0, \sigma_{0}^{2}\right)$ is the additive Gaussian white noise (AWGN) at Bob. And the actual downlink CSI is given by:

$$
\mathbf{h}_{a b d}^{H}=\mathbf{h}_{a b}^{H}+\mathbf{h}_{r b}^{H} \Phi_{2} \mathbf{H}_{a r}
$$

Then the signal-to-interference-plus noise ratio (SINR) at Bob can be derived as

$$
\operatorname{SINR}_{b}=\frac{P_{t}\left|\left(\mathbf{h}_{a b}^{H}+\mathbf{h}_{r b}^{H} \Phi_{2} \mathbf{H}_{a r}\right)\left(\mathbf{h}_{a b}+\mathbf{H}_{a r}^{H} \Phi_{1}^{H} \mathbf{h}_{r b}\right)\right|^{2}}{\sigma^{2}\left\|\mathbf{H}_{a r}^{H} \Phi_{1}^{H} \mathbf{h}_{r b}\right\|_{2}^{2}}
$$

From (3)-(5), we can see that due to the inconsistency between $\Phi_{1}$ and $\Phi_{2}$, the downlink CSI perceived by Alice is misleading and the SINR obtained by Bob is not the optimal.

Further, the received signal at Eve is expressed as

$$
y_{e}=\left(\mathbf{h}_{a e}^{H}+\mathbf{h}_{r e}^{H} \Phi_{2} \mathbf{H}_{a r}\right) \mathbf{w} s+n_{b}
$$

The corresponding SINR is derived as:

$$
\operatorname{SINR}_{e}=\frac{P_{t}\left|\left(\mathbf{h}_{a e}^{H}+\mathbf{h}_{r e}^{H} \Phi_{2} \mathbf{H}_{a r}\right)\left(\mathbf{h}_{a b}+\mathbf{H}_{a r}^{H} \Phi_{1}^{H} \mathbf{h}_{r b}\right)\right|^{2}}{\sigma^{2}\left\|\mathbf{H}_{a r}^{H} \Phi_{1}^{H} \mathbf{h}_{r b}\right\|_{2}^{2}}
$$

Then the system secrecy rate is written as

$R=\left[R_{b}-R_{e}\right]_{+}=\left[\log _{2}\left(1+\operatorname{SINR}_{b}\right)-\log _{2}\left(1+\operatorname{SINR}_{e}\right)\right]_{+}$

where the notation $[x]^{+}=\max \{x, 0\}$ is used.

Therefore, in order to overhear more information, our goal is to minimize the secrecy rate by carefully designing the reflection coefficients $\Phi_{1}$ and $\Phi_{2}$. Hence, the optimization problem can be established as

$$
\begin{aligned}
\min _{\Phi_{1}, \Phi_{2}} R & \\
\text { s.t }\left|\Phi_{1,(i, j)}\right| & = \begin{cases}1, & i=j \\
0, & i \neq j\end{cases} \\
\left|\Phi_{2,(i, j)}\right| & = \begin{cases}1, & i=j \\
0, & i \neq j\end{cases}
\end{aligned}
$$

However, directly designing the reflection coefficient matrix based on instantaneous CSI in problem (9) seems impractical. There exist two reasons. One reason is that during the uplink phase, channel estimation has not yet conducted, and the eavesdropper cannot obtain instantaneous channel information in advance, thus the reflection coefficient matrix $\Phi_{1}$ and $\Phi_{2}$ cannot be designed. The other reason is that Eve eavesdrops on the communication channel passively, and it is relatively difficult to obtain the CSI of each channel in the system, especially for the channel from Alice to Bob. The methods to obtain the global instantaneous CSI is depicted in [15] [16], which need excessive ability requirements. While, after long-term observation, it is reasonable that statistical CSI of each channel can be obtained by Eve. Hence, average secrecy rate as a performance metric is considered and the orginal optimization problem can be transformed to maximum average secrecy rate, which is re-expressed as

$$
\begin{aligned}
& \max _{\Phi_{1} \Phi_{2}} \mathrm{E}\left(R_{b}-R_{e}\right) \\
& =\mathrm{E}\left(\log _{2}\left(1+\operatorname{SINR}_{b}\right)-\log _{2}\left(1+\operatorname{SINR}_{e}\right)\right]_{+} \\
& =\mathrm{E}\left\{\log _{2}\left(\frac{f_{1}\left(\Phi_{1}, \Phi_{2}\right)}{g_{1}\left(\Phi_{1}, \Phi_{2}\right)}\right)\right\} \\
& f_{1}\left(\Phi_{1}, \Phi_{2}\right)=P_{t}\left|\left(\mathbf{h}_{a b}^{H}+\mathbf{h}_{r b}^{H} \Phi_{2} \mathbf{H}_{a r}\right)\left(\mathbf{h}_{a b}+\mathbf{H}_{a r}^{H} \Phi_{1}^{H} \mathbf{h}_{r b}\right)\right|^{2} \\
& +\sigma^{2}\left\|\mathbf{H}_{a r}^{H} \Phi_{1}^{H} \mathbf{h}_{r b}\right\|_{2}^{2} \\
& g_{1}\left(\Phi_{1}, \Phi_{2}\right)=P_{t}\left|\left(\mathbf{h}_{a \mathrm{e}}^{H}+\mathbf{h}_{r e}^{H} \Phi_{2} \mathbf{H}_{a r}\right)\left(\mathbf{h}_{a b}+\mathbf{H}_{a r}^{H} \Phi_{1}^{H} \mathbf{h}_{r b}\right)\right|^{2} \\
& +\sigma^{2}\left\|\mathbf{H}_{a r}^{H} \Phi_{1}^{H} \mathbf{h}_{r b}\right\|_{2}^{2}
\end{aligned}
$$

Note that with statistical CSI, the phase shifts of the uplink phase and the downlink phase can be designed in advance, and be set at the corresponding stage. And when the statistical characteristics of channel changes, the phase shifts need to be updated, which is applicable for quasi-static environment. In this way, the required channel estimation capability for eavesdropper is relatively low.

Further, it is hard to obtain closed-form solutions for problem (10). Fortunately, according to the theorem in [17], an approximate expression can be obtained

$$
\begin{aligned}
& \max _{\Phi_{1}, \Phi_{2}} \mathrm{E}\left\{\log _{2}\left(\frac{f_{1}\left(\Phi_{1}, \Phi_{2}\right)}{g_{1}\left(\Phi_{1}, \Phi_{2}\right)}\right)\right\} \\
\approx & \max _{\Phi_{1}, \Phi_{2}}\left\{\log _{2} \frac{\mathrm{E}\left(f_{1}\left(\Phi_{1}, \Phi_{2}\right)\right)}{\mathrm{E}\left(g_{1}\left(\Phi_{1}, \Phi_{2}\right)\right)}\right\}
\end{aligned}
$$


In this paper, a quasi-static Rician fading environment is assumed. The channel between node $i$ to node $j$ is modeled as $h_{i j}=\sqrt{L_{0} d_{i j}^{-c_{i j}}} g_{i j}$, where $L_{0}$ denotes the path loss at the reference distance $d_{0}=1 \mathrm{~m}, d_{i j}$ denotes the distance from $i$ to $j$, and $c_{i j}$ denotes the corresponding path loss exponent. Besides, the small-scale fading component $g_{i j}$ is assumed to be Rician fading and given by

$$
g_{i j}=\sqrt{\frac{\beta_{i j}}{1+\beta_{i j}}} g_{i j}^{\mathrm{LOS}}+\sqrt{\frac{1}{1+\beta_{i j}}} g_{i j}^{\mathrm{NLOS}}
$$

where $\beta_{i j}$ denotes the Rician factor, $g_{i j}^{\mathrm{LOS}}$ and $g_{i j}^{\mathrm{NLOS}}$ represent the line-of-sight (LoS) and non-LoS (NLoS) components, respectively.

Therefore, the direct channel from Alice to Eve is expressed as

$$
\mathbf{h}_{a \mathrm{e}}=\tilde{\mathbf{h}}_{a \mathrm{e}}+\overline{\mathbf{h}}_{a \mathrm{e}}
$$

where $\overline{\mathbf{h}}_{a \mathrm{e}}$ denotes the LoS component, $\tilde{\mathbf{h}}_{a \mathrm{e}}$ denotes the NLoS component with zero-mean complex Gaussian random variables with variance $\sigma_{a \mathrm{e}}^{2}=\frac{L_{0} d_{a e}^{-c_{a e}}}{1+\beta_{a e}}$.

For other channels, the corresponding LoS and NLoS components can be obtained. Note that since the IRS is deployed vertically high [18] and is deployed near Alice, less scattering environment from Alice to IRS is expected, namely $\mathbf{H}_{a r}=\overline{\mathbf{H}}_{a r}+\tilde{\mathbf{H}}_{a r} \approx \overline{\mathbf{H}}_{a r}$. Then (10b) and (10c) can be rewritten as (14) and (15)

After strict derivation, the expectation operation can be removed and the orginal problem can be rewritten as

$$
\begin{aligned}
& \max _{\Phi_{1}, \Phi_{2}} \log _{2}\left(\frac{\mathrm{E}\left(f_{1}\left(\Phi_{1}, \Phi_{2}\right)\right)}{\mathrm{E}\left(g_{1}\left(\Phi_{1}, \Phi_{2}\right)\right)}\right) \\
& =\max _{\Phi_{1}, \Phi_{2}} \log _{2}\left(\frac{f_{2}\left(\Phi_{1}, \Phi_{2}\right)}{g_{2}\left(\Phi_{1}, \Phi_{2}\right)}\right) \\
& f_{2}\left(\Phi_{1}, \Phi_{2}\right)=P_{t}\left|\left(\overline{\mathbf{h}}_{a b}^{H}+\overline{\mathbf{h}}_{r b}^{H} \Phi_{2} \mathbf{H}_{a r}\right)\left(\overline{\mathbf{h}}_{a b}+\mathbf{H}_{a r}^{H} \Phi_{1}^{H} \overline{\mathbf{h}}_{r b}\right)\right|^{2} \\
& +P_{t} \sigma_{a b}^{2}\left|\left(\overline{\mathbf{h}}_{r b}^{H} \Phi_{1} \mathbf{H}_{a r}+\overline{\mathbf{h}}_{a b}^{H}\right)\right|^{2}+P_{t} \sigma_{a b}^{2}\left|\left(\overline{\mathbf{h}}_{r b}^{H} \Phi_{2} \mathbf{H}_{a r}+\overline{\mathbf{h}}_{a b}^{H}\right)\right|^{2} \\
& +P_{t} \sigma_{r b}^{2}\left|\left(\overline{\mathbf{h}}_{r b}^{H} \Phi_{2} \mathbf{H}_{a r}+\overline{\mathbf{h}}_{a b}^{H}\right) \mathbf{H}_{a r}^{H}\right|^{2}+\sigma^{2}\left|\left(\overline{\mathbf{h}}_{r b}^{H} \Phi_{1} \mathbf{H}_{a r}+\overline{\mathbf{h}}_{a b}^{H}\right)\right|^{2} \\
& +P_{t} \sigma_{r b}^{2}\left|\left(\overline{\mathbf{h}}_{r b}^{H} \Phi_{1} \mathbf{H}_{a r}+\overline{\mathbf{h}}_{a b}^{H}\right) \mathbf{H}_{a r}^{H}\right|^{2}+C_{1} \\
& C_{1}=\sigma^{2} \sigma_{r b}^{2} \operatorname{Tr}\left(\mathbf{H}_{a r} \mathbf{H}_{a r}^{H}\right)+M_{a} \sigma^{2} \sigma_{a b}^{2}+P_{t} M_{a} \sigma_{a b}^{4} \quad(16 \mathrm{~d}) \\
& +2 P_{t} \sigma_{a b}^{2} \sigma_{r b}^{2} \operatorname{Tr}\left(\mathbf{H}_{a r} \mathbf{H}_{a r}^{H}\right)+P_{t} \sigma_{r b}^{4} \operatorname{Tr}\left(\mathbf{H}_{a r} \mathbf{H}_{a r}^{H} \mathbf{H}_{a r} \mathbf{H}_{a r}^{H}\right) \\
& g_{2}\left(\Phi_{1}, \Phi_{2}\right)=P_{t}\left|\left(\overline{\mathbf{h}}_{a e}^{H}+\overline{\mathbf{h}}_{r e}^{H} \Phi_{2} \mathbf{H}_{a r}\right)\left(\overline{\mathbf{h}}_{a b}+\mathbf{H}_{a r}^{H} \Phi_{1}^{H} \overline{\mathbf{h}}_{r b}\right)\right|^{2} \\
& +P_{t} \sigma_{a e}^{2}\left|\left(\overline{\mathbf{h}}_{r b}^{H} \Phi_{1} \mathbf{H}_{a r}+\overline{\mathbf{h}}_{a b}^{H}\right)\right|^{2}+P_{t} \sigma_{a b}^{2}\left|\left(\overline{\mathbf{h}}_{r e}^{H} \Phi_{2} \mathbf{H}_{a r}+\overline{\mathbf{h}}_{a b}^{H}\right)\right|^{2} \\
& +P_{t} \sigma_{r b}^{2}\left|\left(\overline{\mathbf{h}}_{r e}^{H} \Phi_{2} \mathbf{H}_{a r}+\overline{\mathbf{h}}_{a b}^{H}\right) \mathbf{H}_{a r}^{H}\right|^{2}+\sigma^{2}\left|\left(\overline{\mathbf{h}}_{r b}^{H} \Phi_{1} \mathbf{H}_{a r}+\overline{\mathbf{h}}_{a b}^{H}\right)\right|^{2} \\
& +P_{t} \sigma_{r e}^{2}\left|\left(\overline{\mathbf{h}}_{r b}^{H} \Phi_{1} \mathbf{H}_{a r}+\overline{\mathbf{h}}_{a b}^{H}\right) \mathbf{H}_{a r}^{H}\right|^{2}+C_{2} \\
& \quad C_{2}=\sigma^{2} \sigma_{r b}^{2} \operatorname{Tr}\left(\mathbf{H}_{a r} \mathbf{H}_{a r}^{H}\right)+M_{a} \sigma^{2} \sigma_{a b}^{2} \\
& \quad+P_{t} M_{a} \sigma_{a e}^{2} \sigma_{a b}^{2}+P_{t} \sigma_{a e}^{2} \sigma_{r b}^{2} \operatorname{Tr}\left(\mathbf{H}_{a r} \mathbf{H}_{a r}^{H}\right) \\
& \quad+P_{t} \sigma_{a b}^{2} \sigma_{r e}^{2} \operatorname{Tr}\left(\mathbf{H}_{a r} \mathbf{H}_{a r}^{H}\right)+P_{t} \sigma_{r e}^{2} \sigma_{r b}^{2} \operatorname{Tr}_{(\mathbf{H}}\left(\mathbf{H}_{a r} \mathbf{H}_{a r}^{H} \mathbf{H}_{a r} \mathbf{H}_{a r}^{H}\right)
\end{aligned}
$$

Proof: See Appendix A.

Then let $\mathbf{v}_{1}=\left[v_{1,1}, v_{1,2}, \cdots, v_{1, N}\right]^{H}, \quad \mathbf{v}_{2}=$ $\left[v_{2,1}, v_{2,2}, \cdots, v_{2, N}\right]^{H}$, where $v_{1, n}=\Phi_{1,(n, n)}, v_{2, n}=$ $\Phi_{2,(n, n)}, \forall n$. Then we denote $\tilde{\mathbf{v}}_{1}^{H}=e^{j \bar{w}_{1}}\left[\mathbf{v}_{1}^{H}, 1\right]$, $\tilde{\mathbf{v}}_{2}^{H}=e^{j \bar{w}_{2}}\left[\mathbf{v}_{2}^{H}, 1\right]$, where $\bar{w}_{1}, \bar{w}_{2} \in[0,2 \pi]$ is the introduced slack variable without any effect on the optimal solution. By denoting $\mathbf{H}_{a r b}=\operatorname{diag}\left(\overline{\mathbf{h}}_{r b}^{H}\right) \mathbf{H}_{a r}$ and $\mathbf{H}_{a r e}=\operatorname{diag}\left(\overline{\mathbf{h}}_{r e}^{H}\right) \mathbf{H}_{a r}$, we have $\overline{\mathbf{h}}_{r b}^{H} \Phi_{1} \mathbf{H}_{a r}=\mathbf{v}_{1}^{H} \mathbf{H}_{a r b}, \overline{\mathbf{h}}_{r b}^{H} \Phi_{2} \mathbf{H}_{a r}=\mathbf{v}_{2}^{H} \mathbf{H}_{a r b}$. Then (16b) and (16d) can be rewritten as:

$$
\begin{aligned}
& f_{2}\left(\tilde{\mathbf{v}}_{1}, \tilde{\mathbf{v}}_{2}\right)=P_{t}\left|\mathbf{v}_{2}^{H} \overline{\mathbf{H}}_{a b} \overline{\mathbf{H}}_{a b}^{H} \mathbf{v}_{1}\right|^{2}+P_{t} \sigma_{a b}^{2}\left|\mathbf{v}_{1}^{H} \overline{\mathbf{H}}_{a b}^{H}\right|^{2} \\
&+P_{t} \sigma_{a b}^{2}\left|\mathbf{v}_{2}^{H} \overline{\mathbf{H}}_{a b}^{H}\right|^{2}+P_{t} \sigma_{r b}^{2}\left|\mathbf{v}_{2}^{H} \overline{\mathbf{H}}_{a b} \overline{\mathbf{H}}_{a r}^{H}\right|^{2} \\
&+P_{t} \sigma_{r b}^{2}\left|\mathbf{v}_{1}^{H} \overline{\mathbf{H}}_{a b} \overline{\mathbf{H}}_{a r}^{H}\right|^{2}+\sigma^{2}\left|\mathbf{v}_{1}^{H} \overline{\mathbf{H}}_{a b}^{H}\right|^{2}+C_{1} \\
& g_{2}\left(\tilde{\mathbf{v}}_{1}, \tilde{\mathbf{v}}_{2}\right)=P_{t}\left|\mathbf{v}_{2}^{H} \overline{\mathbf{H}}_{a e} \overline{\mathbf{H}}_{a b}^{H} \mathbf{v}_{1}\right|^{2}+P_{t} \sigma_{a e}^{2}\left|\mathbf{v}_{1}^{H} \overline{\mathbf{H}}_{a b}^{H}\right|^{2} \\
& \quad+P_{t} \sigma_{a b}^{2}\left|\mathbf{v}_{2}^{H} \overline{\mathbf{H}}_{a e}^{H}\right|^{2}+P_{t} \sigma_{r b}^{2}\left|\mathbf{v}_{2}^{H} \overline{\mathbf{H}}_{a e} \overline{\mathbf{H}}_{a r}^{H}\right|^{2} \\
& \quad+P_{t} \sigma_{r e}^{2}\left|\mathbf{v}_{1}^{H} \overline{\mathbf{H}}_{a b} \overline{\mathbf{H}}_{a r}^{H}\right|^{2}+\sigma^{2}\left|\mathbf{v}_{1}^{H} \overline{\mathbf{H}}_{a b}^{H}\right|^{2}+C_{2}
\end{aligned}
$$

Further, with the monotonicity of logarithmic function, the orginal problem can be rewritten as

$$
\begin{gathered}
\max _{\tilde{\mathbf{v}}_{1}, \tilde{\mathbf{v}}_{2}} \frac{f_{2}\left(\tilde{\mathbf{v}}_{1}, \tilde{\mathbf{v}}_{2}\right)}{g_{2}\left(\tilde{\mathbf{v}}_{1}, \tilde{\mathbf{v}}_{2}\right)} \\
\text { s.t. }(9 b),(9 c),(16 c),(16 e),(17 a),(17 b)
\end{gathered}
$$

It is worth noting that due to the non-concave fractional constraints as well as the coupling variables and unit-modulus constraints, problem (18) is non-convex and intractable. In the following section, an efficient algorithm is proposed to find a near optimal solution.

\section{B. Problem Solved}

In this section, firstly, alternating optimization is utilized to decompose the orginal problem into two sub-problems. Then, for each sub-problem, the Charnes-Cooper transformation [16] and SDR techniques are utilized to solve each sub-problem respectively.

1) Optimizing $\tilde{\mathbf{v}}_{1}$ for given $\tilde{\mathbf{v}}_{2}$ : Let $\hat{\mathbf{V}}_{1}=\tilde{\mathbf{v}}_{1} \tilde{\mathbf{v}}_{1}^{H}, \hat{\mathbf{V}}_{2}=$ $\tilde{\mathbf{v}}_{2} \tilde{\mathbf{v}}_{2}^{H}$, then we have $\operatorname{Rank}\left(\tilde{\mathbf{V}}_{1}\right)=1, \operatorname{Rank}\left(\tilde{\mathbf{V}}_{2}\right)=1$, and by using the properties of matrix trace, (16b) and (16d) can be rewritten as

$$
\begin{aligned}
& f_{2}\left(\hat{\mathbf{V}}_{1}, \hat{\mathbf{V}}_{2}\right)=P_{t} \sigma_{a b}^{2} \operatorname{Tr}\left(\hat{\mathbf{V}}_{2} \overline{\mathbf{H}}_{a b} \overline{\mathbf{H}}_{a b}^{H}\right)+P_{t} \sigma_{a b}^{2} \operatorname{Tr}\left(\hat{\mathbf{V}}_{1} \overline{\mathbf{H}}_{a b} \overline{\mathbf{H}}_{a b}^{H}\right) \\
& +P_{t} \operatorname{Tr}\left(\hat{\mathbf{V}}_{2} \overline{\mathbf{H}}_{a b} \overline{\mathbf{H}}_{a b}^{H} \hat{\mathbf{V}}_{1} \overline{\mathbf{H}}_{a b} \overline{\mathbf{H}}_{a b}^{H}\right)+P_{t} \sigma_{r b}^{2} \operatorname{Tr}\left(\hat{\mathbf{V}}_{2} \overline{\mathbf{H}}_{a b} \overline{\mathbf{H}}_{a r}^{H} \overline{\mathbf{H}}_{a r} \overline{\mathbf{H}}_{a b}^{H}\right) \\
& +P_{t} \sigma_{r b}^{2} \operatorname{Tr}\left(\hat{\mathbf{V}}_{1} \overline{\mathbf{H}}_{a b} \overline{\mathbf{H}}_{a r}^{H} \overline{\mathbf{H}}_{a r} \overline{\mathbf{H}}_{a b}^{H}\right)+\sigma^{2} \operatorname{Tr}\left(\hat{\mathbf{V}}_{1} \overline{\mathbf{H}}_{a b} \overline{\mathbf{H}}_{a b}^{H}\right)+C_{1}
\end{aligned}
$$

$$
\begin{aligned}
& g_{2}\left(\hat{\mathbf{V}}_{1}, \hat{\mathbf{V}}_{2}\right)=P_{t} \sigma_{a b}^{2} \operatorname{Tr}\left(\hat{\mathbf{V}}_{2} \overline{\mathbf{H}}_{a e} \overline{\mathbf{H}}_{a e}^{H}\right)+P_{t} \sigma_{a e}^{2} \operatorname{Tr}\left(\hat{\mathbf{V}}_{1} \overline{\mathbf{H}}_{a b} \overline{\mathbf{H}}_{a b}^{H}\right) \\
& +P_{t} \operatorname{Tr}\left(\hat{\mathbf{V}}_{2} \overline{\mathbf{H}}_{a e} \overline{\mathbf{H}}_{a b}^{H} \hat{\mathbf{V}}_{1} \overline{\mathbf{H}}_{a b} \overline{\mathbf{H}}_{a e}^{H}\right)+P_{t} \sigma_{r b}^{2} \operatorname{Tr}\left(\hat{\mathbf{V}}_{2} \overline{\mathbf{H}}_{a e} \overline{\mathbf{H}}_{a r}^{H} \overline{\mathbf{H}}_{a r} \overline{\mathbf{H}}_{a e}^{H}\right) \\
& +P_{t} \sigma_{r e}^{2} \operatorname{Tr}\left(\hat{\mathbf{V}}_{1} \overline{\mathbf{H}}_{a b} \overline{\mathbf{H}}_{a r}^{H} \overline{\mathbf{H}}_{a r} \overline{\mathbf{H}}_{a b}^{H}\right)+\sigma^{2} \operatorname{Tr}\left(\hat{\mathbf{V}}_{1} \overline{\mathbf{H}}_{a b} \overline{\mathbf{H}}_{a b}^{H}\right)+C_{2}
\end{aligned}
$$

Since the objective function (18a) is non-convex, CharnesCooper transformation is utilized to transform the fractional operation (18a). First, we introduce auxiliary variables $s_{1}>0$ and define a new matrix $\hat{\mathbf{E}}_{1}=s_{1} \hat{\mathbf{V}}_{1}$. Then we introduce a new function $g_{3}\left(\hat{\mathbf{E}}_{1}, \hat{\mathbf{V}}_{2}\right), f_{3}\left(\hat{\mathbf{E}}_{1}, \hat{\mathbf{V}}_{2}\right)$, which are expressed as

$g_{3}\left(\hat{\mathbf{E}}_{1}, \hat{\mathbf{V}}_{2}\right)=s_{1} \times g_{2}\left(\hat{\mathbf{V}}_{1}, \hat{\mathbf{V}}_{2}\right)$

$$
=P_{t} \sigma_{a b}^{2} \operatorname{Tr}\left(\hat{\mathbf{V}}_{2} \overline{\mathbf{H}}_{a e} \overline{\mathbf{H}}_{a e}^{H}\right)+P_{t} \sigma_{a e}^{2} \operatorname{Tr}\left(\hat{\mathbf{E}}_{1} \overline{\mathbf{H}}_{a b} \overline{\mathbf{H}}_{a b}^{H}\right)
$$

$+P_{t} \operatorname{Tr}\left(\hat{\mathbf{V}}_{2} \overline{\mathbf{H}}_{a e} \overline{\mathbf{H}}_{a b}^{H} \hat{\mathbf{E}}_{1} \overline{\mathbf{H}}_{a b} \overline{\mathbf{H}}_{a e}^{H}\right)+P_{t} \sigma_{r b}^{2} \operatorname{Tr}\left(\hat{\mathbf{V}}_{2} \overline{\mathbf{H}}_{a e} \overline{\mathbf{H}}_{a r}^{H} \overline{\mathbf{H}}_{a r} \overline{\mathbf{H}}_{a e}^{H}\right)$

$+P_{t} \sigma_{r e}^{2} \operatorname{Tr}\left(\hat{\mathbf{E}}_{1} \overline{\mathbf{H}}_{a b} \overline{\mathbf{H}}_{a r}^{H} \overline{\mathbf{H}}_{a r} \overline{\mathbf{H}}_{a b}^{H}\right)+\sigma^{2} \operatorname{Tr}\left(\hat{\mathbf{E}}_{1} \overline{\mathbf{H}}_{a b} \overline{\mathbf{H}}_{a b}^{H}\right)+s_{1} C_{2}$ 


$$
\left.f_{1}\left(\Phi_{1}, \Phi_{2}\right)=P_{t} \mid\left(\left(\overline{\mathbf{h}}_{a b}^{H}+\tilde{\mathbf{h}}_{a b}^{H}\right)+\left(\overline{\mathbf{h}}_{r b}^{H}+\tilde{\mathbf{h}}_{r b}^{H}\right) \Phi_{2} \mathbf{H}_{a r}\right)\right)\left.\left(\left(\overline{\mathbf{h}}_{a b}+\tilde{\mathbf{h}}_{a b}\right)+\mathbf{H}_{a r}^{H} \Phi_{1}^{H}\left(\overline{\mathbf{h}}_{r b}+\tilde{\mathbf{h}}_{r b}\right)\right)\right|^{2}+\sigma^{2}\left\|\mathbf{H}_{a r}^{H} \Phi_{1}^{H}\left(\overline{\mathbf{h}}_{r b}+\tilde{\mathbf{h}}_{r b}\right)\right\|_{2}^{2}
$$

$$
\left.g_{1}\left(\Phi_{1}, \Phi_{2}\right)=P_{t} \mid\left(\left(\overline{\mathbf{h}}_{a \mathrm{e}}^{H}+\tilde{\mathbf{h}}_{a \mathrm{e}}^{H}\right)+\left(\overline{\mathbf{h}}_{r e}^{H}+\tilde{\mathbf{h}}_{r e}^{H}\right) \Phi_{2} \mathbf{H}_{a r}\right)\right)\left.\left(\left(\overline{\mathbf{h}}_{a b}+\tilde{\mathbf{h}}_{a b}\right)+\mathbf{H}_{a r}^{H} \Phi_{1}^{H}\left(\overline{\mathbf{h}}_{r b}+\tilde{\mathbf{h}}_{r b}\right)\right)\right|^{2}+\sigma^{2}\left\|\mathbf{H}_{a r}^{H} \Phi_{1}^{H}\left(\overline{\mathbf{h}}_{r b}+\tilde{\mathbf{h}}_{r b}\right)\right\|_{2}^{2}
$$

$f_{3}\left(\hat{\mathbf{E}}_{1}, \hat{\mathbf{V}}_{2}\right)=s_{1} \times f_{2}\left(\hat{\mathbf{V}}_{1}, \hat{\mathbf{V}}_{2}\right)$

$=P_{t} \sigma_{a b}^{2} \operatorname{Tr}\left(\hat{\mathbf{V}}_{2} \overline{\mathbf{H}}_{a b} \overline{\mathbf{H}}_{a b}^{H}\right)+P_{t} \sigma_{a b}^{2} \operatorname{Tr}\left(\hat{\mathbf{E}}_{1} \overline{\mathbf{H}}_{a b} \overline{\mathbf{H}}_{a b}^{H}\right)$

$+P_{t} \operatorname{Tr}\left(\hat{\mathbf{V}}_{2} \overline{\mathbf{H}}_{a b} \overline{\mathbf{H}}_{a b}^{H} \hat{\mathbf{E}}_{1} \overline{\mathbf{H}}_{a b} \overline{\mathbf{H}}_{a b}^{H}\right)+P_{t} \sigma_{r b}^{2} \operatorname{Tr}\left(\hat{\mathbf{V}}_{2} \overline{\mathbf{H}}_{a b} \overline{\mathbf{H}}_{a r}^{H} \overline{\mathbf{H}}_{a r} \overline{\mathbf{H}}\right.$

$+P_{t} \sigma_{r b}^{2} \operatorname{Tr}\left(\hat{\mathbf{E}}_{1} \overline{\mathbf{H}}_{a b} \overline{\mathbf{H}}_{a r}^{H} \overline{\mathbf{H}}_{a r} \overline{\mathbf{H}}_{a b}^{H}\right)+\sigma^{2} \operatorname{Tr}\left(\hat{\mathbf{E}}_{1} \overline{\mathbf{H}}_{a b} \overline{\mathbf{H}}_{a b}^{H}\right)+s_{1} C_{1}$

Then problem (18) is equivalently transformed as:

$$
\begin{array}{ll} 
& \max _{\hat{\mathbf{E}}_{1}} f_{3}\left(\hat{\mathbf{E}}_{1}, \hat{\mathbf{V}}_{2}\right) \\
\text { s.t. } & g_{3}\left(\hat{\mathbf{E}}_{1}, \hat{\mathbf{V}}_{2}\right)=1 \\
& \hat{\mathbf{E}}_{1(n, n)}=s_{1}, n=1,2, \cdots(N+1) \\
& \operatorname{Rank}\left(\hat{\mathbf{E}}_{1}\right)=1
\end{array}
$$

Note that problem (23) is non-convex due to the constraint (23d). Then, the Rank-1 constraint is firstly discarded and problem (23) is transformed to a convex semidefinite programming problem with linear constraints and can be efficiently solved via the CVX solver. Since the constraint of Rank-1 is relaxed in problem (23), it is necessary to verify whether the rank of the obtained solution satisfies Rank-1. If the obtained solution is not Rank-1, gaussian randomization is applied for recovering vector approximately [9].

2) Optimizing $\tilde{\mathbf{v}}_{2}$ for given $\tilde{\mathbf{v}}_{2}$ : Similarly, slack variable $s_{2}>0$ is introduced, which satisfies $s_{2} \times g_{2}\left(\hat{\mathbf{V}}_{1}, \hat{\mathbf{V}}_{2}\right)=1$. And new matrix $\hat{\mathbf{E}}_{2}=s_{2} \hat{\mathbf{V}}_{2}$ is defined. Then new functions $g_{4}\left(\hat{\mathbf{V}}_{1}, \hat{\mathbf{E}}_{2}\right), f_{4}\left(\hat{\mathbf{V}}_{1}, \hat{\mathbf{E}}_{2}\right)$ are constructed as follows

$g_{4}\left(\hat{\mathbf{V}}_{1}, \hat{\mathbf{E}}_{2}\right)=s_{2} \times g_{2}\left(\hat{\mathbf{V}}_{1}, \hat{\mathbf{V}}_{2}\right)$

$=P_{t} \sigma_{a b}^{2} \operatorname{Tr}\left(\hat{\mathbf{E}}_{2} \overline{\mathbf{H}}_{a b} \overline{\mathbf{H}}_{a e}^{H}\right)+P_{t} \sigma_{r b}^{2} \operatorname{Tr}\left(\hat{\mathbf{E}}_{2} \overline{\mathbf{H}}_{a e} \overline{\mathbf{H}}_{a r}^{H} \overline{\mathbf{H}}_{a r} \overline{\mathbf{H}}_{a e}^{H}\right)$

$+P_{t} \operatorname{Tr}\left(\hat{\mathbf{E}}_{2} \overline{\mathbf{H}}_{a e} \overline{\mathbf{H}}_{a b}^{H} \hat{\mathbf{V}}_{1} \overline{\mathbf{H}}_{a b} \overline{\mathbf{H}}_{a e}^{H}\right)+P_{t} \sigma_{a e}^{2} \operatorname{Tr}\left(\hat{\mathbf{V}}_{1} \overline{\mathbf{H}}_{a b} \overline{\mathbf{H}}_{a b}^{H}\right)$

$+P_{t} \sigma_{r e}^{2} \operatorname{Tr}\left(\hat{\mathbf{V}}_{1} \overline{\mathbf{H}}_{a b} \overline{\mathbf{H}}_{a r}^{H} \overline{\mathbf{H}}_{a r} \overline{\mathbf{H}}_{a b}^{H}\right)+\sigma^{2} \operatorname{Tr}\left(\hat{\mathbf{V}}_{1} \overline{\mathbf{H}}_{a b} \overline{\mathbf{H}}_{a b}^{H}\right)+s_{2} C_{1}$

$f_{4}\left(\hat{\mathbf{V}}_{1}, \hat{\mathbf{E}}_{2}\right)=s_{2} \times f_{2}\left(\hat{\mathbf{V}}_{1}, \hat{\mathbf{V}}_{2}\right)$

$=P_{t} \sigma_{a b}^{2} \operatorname{Tr}\left(\hat{\mathbf{E}}_{2} \overline{\mathbf{H}}_{a b} \overline{\mathbf{H}}_{a b}^{H}\right)+P_{t} \sigma_{r b}^{2} \operatorname{Tr}\left(\hat{\mathbf{E}}_{2} \overline{\mathbf{H}}_{a b} \overline{\mathbf{H}}_{a r}^{H} \overline{\mathbf{H}}_{a r} \overline{\mathbf{H}}_{a e}^{H}\right)$

$+P_{t} \operatorname{Tr}\left(\hat{\mathbf{E}}_{2} \overline{\mathbf{H}}_{a b} \overline{\mathbf{H}}_{a b}^{H} \hat{\mathbf{V}}_{1} \overline{\mathbf{H}}_{a b} \overline{\mathbf{H}}_{a b}^{H}\right)+P_{t} \sigma_{a b}^{2} \operatorname{Tr}\left(\hat{\mathbf{V}}_{1} \overline{\mathbf{H}}_{a b} \overline{\mathbf{H}}_{a b}^{H}\right)$

$+P_{t} \sigma_{r b}^{2} \operatorname{Tr}\left(\hat{\mathbf{V}}_{1} \overline{\mathbf{H}}_{a b} \overline{\mathbf{H}}_{a r}^{H} \overline{\mathbf{H}}_{a r} \overline{\mathbf{H}}_{a b}^{H}\right)+\sigma^{2} \operatorname{Tr}\left(\hat{\mathbf{V}}_{1} \overline{\mathbf{H}}_{a b} \overline{\mathbf{H}}_{a b}^{H}\right)+s_{1} C_{1}$

Then the orginal problem is equivalently transformed as:

$$
\begin{aligned}
& \max _{\hat{\mathbf{E}}_{1}} f_{4}\left(\hat{\mathbf{V}}_{1}, \hat{\mathbf{E}}_{2}\right) \\
& \text { s.t. } g_{4}\left(\hat{\mathbf{V}}_{1}, \hat{\mathbf{E}}_{2}\right)=1 \\
& \hat{\mathbf{E}}_{2(n, n)}=s_{2}, n=1,2, \cdots(N+1) \\
& \quad \operatorname{Rank}\left(\hat{\mathbf{E}}_{2}\right)=1
\end{aligned}
$$

Similarly, by dropping Rank-1 constraint (26d), problem (26) can be efficiently solved via the CVX solver. And then IGaussian randomization method is utilized to recover $\tilde{\mathbf{v}}_{2}$.

3) Overall Algorithm: To summarize, the outline of solving problem (18) is given in Algorithm 1, where $L$ denotes the maximum iteration number.

\begin{tabular}{l}
\hline Algorithm 1 proposed algorithm for solving problem (18) \\
\hline 1. Initialize the phase shifts of the IRS as $\tilde{\mathbf{v}}_{1}^{*(0)}=$ \\
$e^{j \bar{w}_{1}}\left[\begin{array}{c}\mathbf{v}_{1}^{(0)} \\
1\end{array}\right], \tilde{\mathbf{v}}_{2}^{*(0)}=e^{j \bar{w}_{2}}\left[\begin{array}{c}\mathbf{v}_{2}^{(0)} \\
1\end{array}\right]$ with random value \\
$\bar{w}_{1}, \bar{w}_{2} \in[0,2 \pi]$, and set $l=1 ;$ \\
2. Repeat \\
3. Solve (23) for given $\tilde{\mathbf{v}}_{2}^{(l-1)}$, and obtain the solution as \\
$\tilde{\mathbf{v}}_{1}^{(l)}$. \\
4. Solve (26) for given $\tilde{\mathbf{v}}_{1}^{(l)}$, and obtain the solution as $\tilde{\mathbf{v}}_{2}^{(l)}$. \\
5. Update $l=l+1$. \\
6. Until the fractional decrease of the objective value is \\
below the error $\varepsilon$ or the iteration number meet $l=L$. \\
\hline
\end{tabular}

Convergence Analysis: For the $l$-th alternating iteration, denote $R\left(\tilde{\mathbf{v}}_{1}^{(l)}, \tilde{\mathbf{v}}_{2}^{(l)}\right)$ as the objective function value, where $\left(\tilde{\mathbf{v}}_{1}^{(l)}, \tilde{\mathbf{v}}_{2}^{(l)}\right)$ is the feasible solution. Then for the $(l+1)$-th iteration, $\left(\tilde{\mathbf{v}}_{1}^{(l+1)}, \tilde{\mathbf{v}}_{2}^{(l)}\right)$ is the feasible solution of problem (23), and $\left(\tilde{\mathbf{v}}_{1}^{(l+1)}, \tilde{\mathbf{v}}_{2}^{(l+1)}\right)$ is the feasible solution of problem (26). Then, we have

$$
R\left(\tilde{\mathbf{v}}_{1}^{(l)}, \tilde{\mathbf{v}}_{2}^{(l)}\right) \stackrel{(a)}{\geq} R\left(\tilde{\mathbf{v}}_{1}^{(l+1)}, \tilde{\mathbf{v}}_{2}^{(l)}\right) \stackrel{(b)}{\geq} R\left(\tilde{\mathbf{v}}_{1}^{(l+1)}, \tilde{\mathbf{v}}_{2}^{(l+1)}\right)
$$

where inequality (a) holds due to the fact that problem (23) is solved optimally in step 3, equality (b) holds due to step 4. This indicates that the object value given in the $(l+1)$-th iteration is not larger than that in the $l$-th iteration. That is to say, after each iteration, the object value is non-increasing. Furthermore, along with the QoS constraint, the secrecy rate is lower bounded and thus it must guarantee to converge after some iterations.

Complexity Analysis: The main complexity of Algorithm 2 lies on the Step 3, 4. For Step 3, the complexity for solving (14) using the interior-point method, which is denoted as $\mathcal{O}_{1}$, is determined by the number and size of variables (design variables and slack variables) and constraints (PSD constraints and slack constraints), which is summarized in Table I. Since the same form between (23) and (26), the complexity of Step 4 is equal to that of Step 3. Furthermore, the major complexity of Algorithm 2 is given by $2 \times I_{a o} \times \mathcal{O}_{1}$, where $I_{a o}$ denotes the alternating iteration numbers. 
TABLE I: Computational complexity analysis for the proposed algorithm

\begin{tabular}{ccccc}
\hline \multirow{2}{*}{ Algorithm } & \multicolumn{2}{c}{ Variables } & $\begin{array}{c}\text { PSD } \\
\text { constraints }\end{array}$ & $\begin{array}{c}\text { Slack } \\
\text { constraints }\end{array}$ \\
\cline { 2 - 5 } complexity & $\begin{array}{c}\text { design varables } \\
(\text { size }, \text { number })\end{array}$ & $\begin{array}{c}\text { Slack variables } \\
(\text { size, number })\end{array}$ & $\begin{array}{c}\text { (size,number) } \\
\text { (number) }\end{array}$ \\
\hline Step3 & $(N \times N, 1)$ & $(1 \times 1,1)$ & $(N \times N, 1)$ & $(N+2)$ \\
\hline
\end{tabular}

\section{NUMERICAL RESULTS}

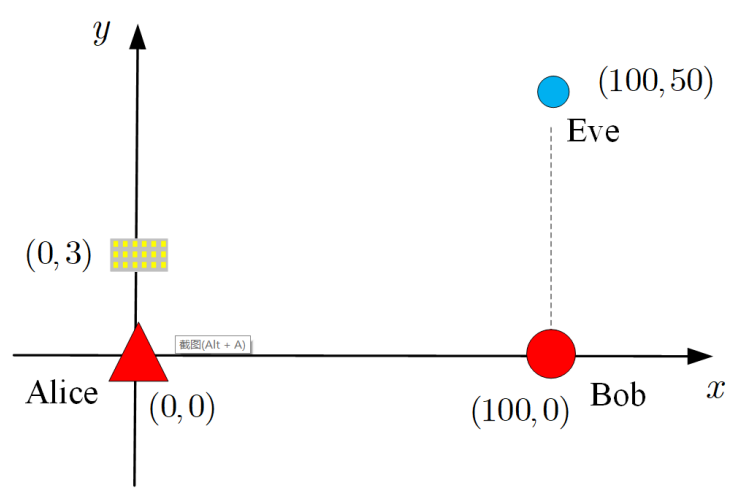

Fig. 3: Simulation Setup

In this section, we present numerical results to validate the performance of the proposed scheme. Simulation setups are shown in Fig. 2, where Alice, Bob, IRS, Eve are located at $(0,0),(100,0),(0,3),(100,50)$ in meter $(\mathrm{m})$, respectively. Considering that IRS is deployed vertically high [18], a less scattering environment is expected and thus we set $\beta_{a r}=\infty$, $c_{a r}=2.5, c_{r e}=c_{r b}=3, \beta_{r b}=10, \beta_{r e}=5$. The rest parameters are listed as follows: $c_{a e}=c_{a b}=3, \beta_{a b}=10$, $\sigma_{0}^{2}=-80 \mathrm{dBm}, L_{0}=-30 \mathrm{~dB}$. The iterative threshold $\varepsilon=0.001$, and the maximum iteration number $L=8$. The following simulation results are achieved by averaging over 500 randomly channel realizations.

Fig. 3 shows the convergence behavior of our proposed algorithm when the number of reflecting elements at the IRS is 20 and 40, respectively. We can observe that with the increase of iteration numbers, the system secrecy rate gradually decreases. After about 6 iterations, the object values all reach stable values, which validates the convergence analysis given in Section III.C.

In order to evaluate the advantage brought by the IRS, we compare our proposed scheme (Active IRS) with the following three benchmark schemes:

Scheme 1: Without IRS. In this case, Eve passively wiretap information without the aid of the IRS.

Scheme 2: Jamming assisted pilot spoofing scheme (Pilot Jam). In this case, Eve sends jamming noise when Bob sends the uplink pilot to Alice as shown in [2]. The jamming pilot power is set to 0.01 of the transmit power by Alice.

Scheme 3: Passive IRS. In this case, the phase shifts at the IRS keep constanst during the uplink phase and downlink phase. And the phase shifts is designed cooperating with Eve to minimize the secrecy rate. However, in this scheme, the probed CSI for Alice is not misleaded by the IRS, and it is the only selected strategy for FDD system.

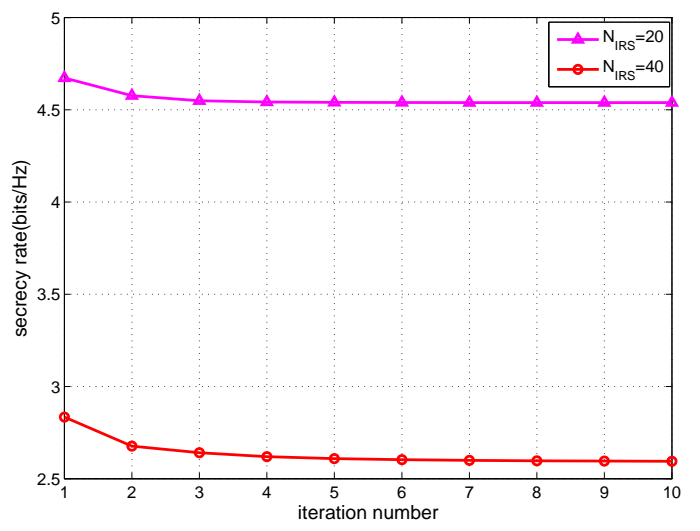

Fig. 4: Convergence behavior verus the iteration numbers of alternating optimization $\left(M_{e}=10, \beta_{a b}=10, \beta_{a e}=5\right)$

Fig. 4 shows the system secrecy rate verus different transmit power sent by Alice. As the transmit power increases, the secrecy rate increases gradually. Meanwhile, we can observe that, under the same condition, the proposed scheme is almost equal to Scheme 2 with Jamming and significantly outperforms the other two benchmark schemes. The reason that the scheme is superior to the scheme 1 and 3 can be concluded from problem (17). By carefully designing reflection coefficients, the misleaded beam sent by Alice can be intendly aligned to Eve during the uplink phase, and during the downlink phase, the received signal by Eve can be intendly enhanced. Moreover, compared with Scheme 2, no addition power is consumed by Eve.

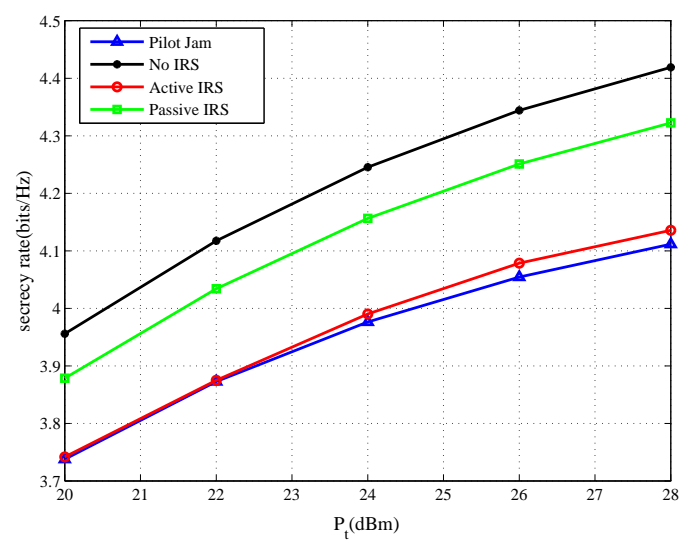

Fig. 5: the secrecy rate verus transmit power $\left(N_{\text {IRS }}=\right.$ $\left.20, M_{e}=10, \beta_{a b}=10, \beta_{a e}=5\right)$

The performance gains verus the number of transmit antennas at Alice is plotted in Fig.5. It can be seen that as the 
number of transmit antennas increases, the system secrecy rate increases gradually. The reason is that beamforming technique can be effectively utilized with more transmit antennas. From Fig.5, we can see that under the same constraint, when the number of transmit antennas is relatively low, our proposed scheme achieves the best performance, and when the number of transmit antennas are increases to a high level, scheme 2 achieves the best performance, which illustrates that jamming assisted pilot spoofing poses more disadvantage to secure communication. Moreover, compared with passive eavesdropping scheme, the performance of our proposed scheme difference decreases more as increasing the number of transmit antennas, which illustrates that due to pilot spoofing, more beam leakage although multiple antennas are utilized.

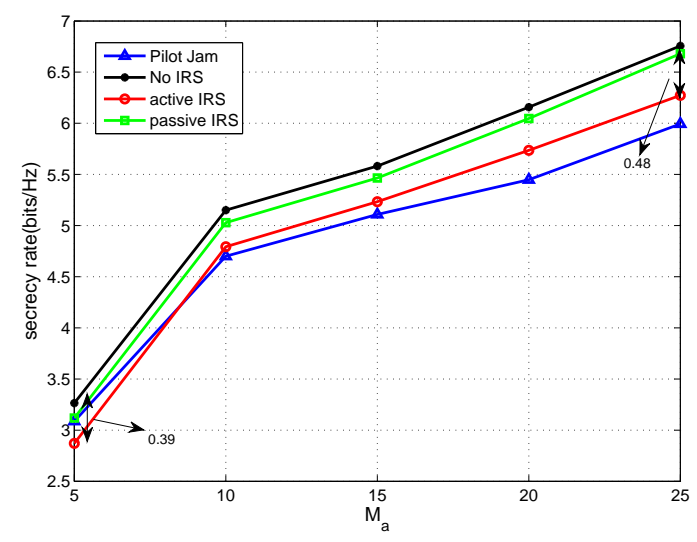

Fig. 6: the secrecy rate verus the number of transmit antennas at Alice $\left(N_{\mathrm{IRS}}=20, \beta_{a b}=10, M_{a}=10, \beta_{a e}=5\right)$

In Fig.8, we further shows the performance gains verus the number of reflecting elements. It can be seen that for pasive IRS and active IRS assisted scheme, as the number of reflection elements increases, the system secrecy rate decreases gradually. The reason is that IRS with larger elements can provide more adjustable dimension for inequality (18a). Moreover, form Fig 5-8, we can observe that passive-assisted scheme poses less damage than active-assisted scheme, which illustrates that our proposed scheme poses more damage to TDD system than FDD system and should be paid more attention to this attack. In fact, the number of reflecting elements can reach 100 or more at a low cost [?], which could bring more secure damage with less power.

\section{Conclusion And Future Work}

In this paper, we have presented an investigation of IRSassisted pilot spoofing scheme. Firstly, the control strategy of the IRS is proposed. By changing the phase shifts during uplink phase and downlink phase, the reciprocity between uplink and downlink disappears and the secure beamforming vector shifts, which leads to signal leakage. Then, the minimum average safety rate problem based on statistical CSI is established by carefully designing the phase shifts. With alternating optimization algorithm and Charnes-Cooper transformation technique, a sub-optimal solution is proposed.

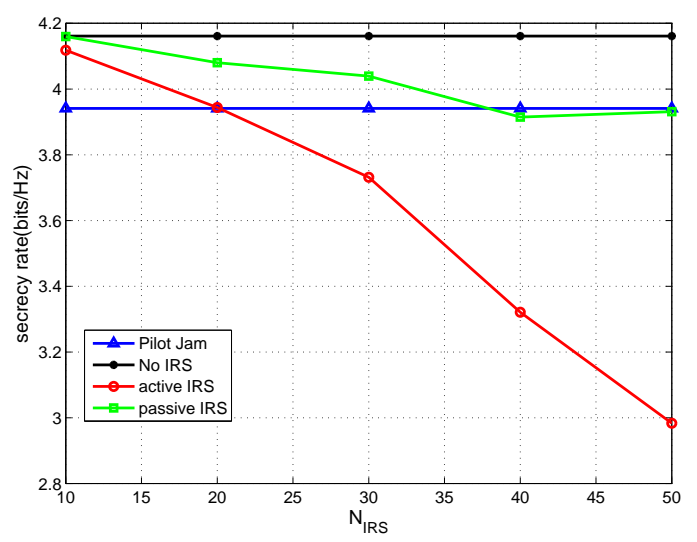

Fig. 7: the minimum interference power verus the number of reflecting elements $\left(M_{a}=10, \beta_{a b}=10, \beta_{a e}=5\right)$

Finally, simulation results show that our scheme can seriously affect the security performance of the TDD systems without energy consumption. If the IRS is not utilized by the internal users properly, it will bring serious threat. Therefore, it is worth paying attention to studying the effective countermeasures against pilot spoofing attack in future study.

\section{APPENDIX A}

\section{PROOF OF PROPOSITION 1}

In this appendix, the transformation of expected operation is proved here. Due to the similar form of the denominator $\mathrm{E}\left(g_{1}\left(\Phi_{1}, \Phi_{2}\right)\right)$ and the numerator $\mathrm{E}\left(f_{1}\left(\Phi_{1}, \Phi_{2}\right)\right)$ in (11), the denominator $\mathrm{E}\left(g_{1}\left(\Phi_{1}, \Phi_{2}\right)\right)$ in (11) is firstly transformed.

$$
\begin{aligned}
& g_{2}\left(\Phi_{1}, \Phi_{2}\right)=\mathrm{E}\left(g_{1}\left(\Phi_{1}, \Phi_{2}\right)\right) \\
& =\mathrm{E}\left(\sigma^{2}\left\|\mathbf{H}_{a r}^{H} \Phi_{1}^{H} \mathbf{h}_{r b}\right\|_{2}^{2}\right. \\
& \left.\quad+P_{t}\left|\left(\mathbf{h}_{a \mathrm{e}}^{H}+\mathbf{h}_{r e}^{H} \Phi_{2} \mathbf{H}_{a r}\right)\left(\mathbf{h}_{a b}+\mathbf{H}_{a r}^{H} \Phi_{1}^{H} \mathbf{h}_{r b}\right)\right|^{2}\right)
\end{aligned}
$$

Let $q_{1}=\left|\left(\mathbf{h}_{a e}^{H}+\mathbf{h}_{r e}^{H} \mathbf{A}\right)\left(\mathbf{h}_{a b}+\mathbf{B}^{H} \mathbf{h}_{r b}\right)\right|^{2}, q_{2}=$ $\left\|\mathbf{B}^{H}\left(\overline{\mathbf{h}}_{r b}+\tilde{\mathbf{h}}_{r b}\right)\right\|_{2}^{2}$, where $\mathbf{A}=\Phi_{2} \mathbf{H}_{a r}, \mathbf{B}=\Phi_{1} \mathbf{H}_{a r}$, then $q_{1}$ can be firstly expanded as

$$
\begin{aligned}
& q_{1}=\left|\left(\mathbf{h}_{a e}^{H}+\mathbf{h}_{r e}^{H} \mathbf{A}\right)\left(\mathbf{h}_{a b}+\mathbf{B}^{H} \mathbf{h}_{r b}\right)\right|^{2} \\
& =\left|\mathbf{h}_{a e}^{H} \mathbf{h}_{a b}+\mathbf{h}_{a e}^{H} \mathbf{B}^{H} \mathbf{h}_{r b}+\mathbf{h}_{r e}^{H} \mathbf{A} \mathbf{h}_{a b}+\mathbf{h}_{r e}^{H} \mathbf{A} \mathbf{B}^{H} \mathbf{h}_{r b}\right|^{2} \\
& =\underbrace{\mathbf{h}_{a e}^{H} \mathbf{h}_{a b} \mathbf{h}_{a b}^{H} \mathbf{h}_{a e}}_{p_{1}}+\underbrace{\mathbf{h}_{a e}^{H} \mathbf{h}_{a b} \mathbf{h}_{r b}^{H} \mathbf{B} \mathbf{h}_{a e}}_{p_{2}}+\underbrace{\mathbf{h}_{a e}^{H} \mathbf{h}_{a b} \mathbf{h}_{a b}^{H} \mathbf{A}^{H} \mathbf{h}_{r e}}_{p_{3}} \\
& +\underbrace{\mathbf{h}_{a e}^{H} \mathbf{h}_{a b} \mathbf{h}_{r b}^{H} \mathbf{B} A^{H} \mathbf{h}_{r e}}_{p_{4}}+\underbrace{\mathbf{h}_{a e}^{H} \mathbf{B}^{H} \mathbf{h}_{r b} \mathbf{h}_{a b}^{H} \mathbf{h}_{a e}}_{p_{5}} \\
& +\underbrace{\mathbf{h}_{a e}^{H} \mathbf{B}^{H} \mathbf{h}_{r b} \mathbf{h}_{r b}^{H} \mathbf{B} \mathbf{h}_{a e}}_{p_{6}}+\underbrace{\mathbf{h}_{a e}^{H} \mathbf{B}^{H} \mathbf{h}_{r b} \mathbf{h}_{a b}^{H} \mathbf{A}^{H} \mathbf{h}_{r e}}_{p_{7}} \\
& +\underbrace{\mathbf{h}_{a e}^{H} \mathbf{B}^{H} \mathbf{h}_{r b} \mathbf{h}_{r b}^{H} \mathbf{B} A^{H} \mathbf{h}_{r e}}_{p_{8}}+\underbrace{\mathbf{h}_{r e}^{H} \mathbf{A} \mathbf{h}_{a b} \mathbf{h}_{a b}^{H} \mathbf{h}_{a e}}_{p_{9}} \\
& +\underbrace{\mathbf{h}_{r e}^{H} \mathbf{A} \mathbf{h}_{a b} \mathbf{h}_{r b}^{H} \mathbf{B} \mathbf{h}_{a e}}_{p_{10}}+\underbrace{\mathbf{h}_{r e}^{H} \mathbf{A} \mathbf{h}_{a b} \mathbf{h}_{a b}^{H} \mathbf{A}^{H} \mathbf{h}_{r e}}_{p_{11}} \\
& +\underbrace{\mathbf{h}_{r e}^{H} \mathbf{A} \mathbf{h}_{a b} \mathbf{h}_{r b}^{H} \mathbf{B} A^{H} \mathbf{h}_{r e}}_{p_{12}}+\underbrace{\mathbf{h}_{r e}^{H} \mathbf{A} \mathbf{B}^{H} \mathbf{h}_{r b} \mathbf{h}_{a b}^{H} \mathbf{h}_{a e}}_{p_{13}}
\end{aligned}
$$




$$
\begin{aligned}
& +\underbrace{\mathbf{h}_{r e}^{H} \mathbf{A} \mathbf{B}^{H} \mathbf{h}_{r b} \mathbf{h}_{r b}^{H} \mathbf{B} \mathbf{h}_{a e}}_{p_{14}}+\underbrace{\mathbf{h}_{r e}^{H} \mathbf{A} \mathbf{B}^{H} \mathbf{h}_{r b} \mathbf{h}_{a b}^{H} \mathbf{A}^{H} \mathbf{h}_{r e}}_{p_{15}} \\
& +\underbrace{\mathbf{h}_{r e}^{H} \mathbf{A} \mathbf{B}^{H} \mathbf{h}_{r b} \mathbf{h}_{r b}^{H} \mathbf{B} A^{H} \mathbf{h}_{r e}}_{p_{16}}
\end{aligned}
$$

(28) is consisted of 16 subitems, and we can transform each term one by one. In order to effectively illustrate the transformation process, we take the most complex term $p_{16}$ as an example. Then each channel vector is expanded as the sum of the LoS component and NLoS component and $p_{16}$ can be rewritten as

$$
\begin{aligned}
\mathrm{E} & \left(p_{16}\right) \\
& =\mathrm{E}\left\{\left(\overline{\mathbf{h}}_{r e}^{H}+\tilde{\mathbf{h}}_{r e}^{H}\right) \mathbf{A} \mathbf{B}^{H}\left(\overline{\mathbf{h}}_{r b}+\tilde{\mathbf{h}}_{r b}\right)\left(\overline{\mathbf{h}}_{r b}^{H}+\tilde{\mathbf{h}}_{r b}^{H}\right) \mathbf{B} A^{H}\left(\overline{\mathbf{h}}_{r e}+\tilde{\mathbf{h}}_{r e}\right)\right\} \\
& =\underbrace{\mathrm{E}\left(\overline{\mathbf{h}}_{r e}^{H} \mathbf{A} \mathbf{B}^{H} \overline{\mathbf{h}}_{r b} \overline{\mathbf{h}}_{r b}^{H} \mathbf{B} A^{H} \overline{\mathbf{h}}_{r e}\right)}_{t_{1}}+\underbrace{\mathrm{E}\left(\overline{\mathbf{h}}_{r e}^{H} \mathbf{A} \mathbf{B}^{H} \overline{\mathbf{h}}_{r b} \overline{\mathbf{h}}_{r b}^{H} \mathbf{B} A^{H} \tilde{\mathbf{h}}_{r e}\right)}_{t_{2}} \\
& +\underbrace{\mathrm{E}\left(\overline{\mathbf{h}}_{r e}^{H} \mathbf{A} \mathbf{B}^{H} \overline{\mathbf{h}}_{r b} \tilde{\mathbf{h}}_{r b}^{H} \mathbf{B} A^{H} \overline{\mathbf{h}}_{r e}\right)}_{t_{t}}+\underbrace{\mathrm{E}\left(\overline{\mathbf{h}}_{r e}^{H} \mathbf{A} \mathbf{B}^{H} \overline{\mathbf{h}}_{r b} \tilde{\mathbf{h}}_{r b}^{H} \mathbf{B} A^{H} \tilde{\mathbf{h}}_{r e}\right)}_{t_{t}} \\
& +\underbrace{\mathrm{E}\left(\overline{\mathbf{h}}_{r e}^{H} \mathbf{A} \mathbf{B}^{H} \tilde{\mathbf{h}}_{r b} \overline{\mathbf{h}}_{r b}^{H} \mathbf{B} A^{H} \overline{\mathbf{h}}_{r e}\right)}_{t_{t}}+\underbrace{\mathrm{E}\left(\overline{\mathbf{h}}_{r e}^{H} \mathbf{A} \mathbf{B}^{H} \tilde{\mathbf{h}}_{r b} \overline{\mathbf{h}}_{r b}^{H} \mathbf{B} A^{H} \tilde{\mathbf{h}}_{r e}\right)}_{t_{t}} \\
& +\underbrace{\mathrm{E}\left(\overline{\mathbf{h}}_{r e}^{H} \mathbf{A} \mathbf{B}^{H} \tilde{\mathbf{h}}_{r b} \tilde{\mathbf{h}}_{r b}^{H} \mathbf{B} A^{H} \overline{\mathbf{h}}_{r e}\right)}_{t_{10}}+\underbrace{\mathrm{E}\left(\overline{\mathbf{h}}_{r e}^{H} \mathbf{A} \mathbf{B}^{H} \tilde{\mathbf{h}}_{r b} \tilde{\mathbf{h}}_{r b}^{H} \mathbf{B} A^{H} \tilde{\mathbf{h}}_{r e}\right)}_{t_{16}} \\
& +\underbrace{\mathrm{E}\left(\tilde{\mathbf{h}}_{r e}^{H} \mathbf{A} \mathbf{B}^{H} \overline{\mathbf{h}}_{r b} \tilde{\mathbf{h}}_{r b}^{H} \mathbf{B} A^{H} \overline{\mathbf{h}}_{r e}\right)}_{\left.t_{13} \overline{\mathbf{h}}_{r b} \overline{\mathbf{h}}_{r b}^{H} \mathbf{B} A^{H} \overline{\mathbf{h}}_{r e}\right)}+\underbrace{\mathrm{E}\left(\tilde{\mathbf{h}}_{r e}^{H} \mathbf{A} \mathbf{B}^{H} \overline{\mathbf{h}}_{r b} \overline{\mathbf{h}}_{r b}^{H} \mathbf{B} A^{H} \tilde{\mathbf{h}}_{r e}\right)}_{t_{12}} \\
& +\underbrace{\mathrm{E}\left(\tilde{\mathbf{h}}_{r e}^{H} \mathbf{A} \mathbf{B}^{H} \tilde{\mathbf{h}}_{r b} \overline{\mathbf{h}}_{r b}^{H} \mathbf{B} A^{H} \overline{\mathbf{h}}_{r e}\right)}_{t_{14}\left(\tilde{\mathbf{h}}_{r e}^{H} \mathbf{A} \mathbf{B}^{H} \overline{\mathbf{h}}_{r b} \tilde{\mathbf{h}}_{r b}^{H} \mathbf{B} A^{H} \tilde{\mathbf{h}}_{r e}\right)}+\underbrace{\mathrm{E}\left(\tilde{\mathbf{h}}_{r e}^{H} \mathbf{A} \mathbf{B}^{H} \tilde{\mathbf{h}}_{r b} \overline{\mathbf{h}}_{r b}^{H} \mathbf{B} A^{H} \tilde{\mathbf{h}}_{r e}\right)}_{t_{13}} \\
+ & \underbrace{\mathrm{E}\left(\tilde{\mathbf{h}}_{r e}^{H} \mathbf{A B} \mathbf{B}^{H} \tilde{\mathbf{h}}_{r b} \tilde{\mathbf{h}}_{r b}^{H} \mathbf{B} A^{H} \tilde{\mathbf{h}}_{r e}\right)}_{\tilde{t}_{r e}\left(\tilde{\mathbf{h}}_{r e}^{H} \mathbf{A B} \mathbf{B}^{H} \tilde{\mathbf{h}}_{r b} \tilde{\mathbf{h}}_{r b}^{H} \mathbf{B} A^{H} \overline{\mathbf{h}}_{r e}\right)}
\end{aligned}
$$

Then, for each term in (30), we calculate the expectation separately. First, we can have

$$
\begin{gathered}
E\left(t_{1}\right)=E\left(\overline{\mathbf{h}}_{r e}^{H} \mathbf{A} \mathbf{B}^{H} \overline{\mathbf{h}}_{r b} \overline{\mathbf{h}}_{r b}^{H} \mathbf{B} A^{H} \overline{\mathbf{h}}_{r e}\right) \\
\stackrel{(a)}{=} \overline{\mathbf{h}}_{r e}^{H} \mathbf{A} \mathbf{B}^{H} \overline{\mathbf{h}}_{r b} \overline{\mathbf{h}}_{r b}^{H} \mathbf{B} A^{H} \overline{\mathbf{h}}_{r e} \\
E\left(t_{2}\right)=E\left(\overline{\mathbf{h}}_{r e}^{H} \mathbf{A} \mathbf{B}^{H} \overline{\mathbf{h}}_{r b} \overline{\mathbf{h}}_{r b}^{H} \mathbf{B} A^{H} \tilde{\mathbf{h}}_{r e}\right) \stackrel{(b)}{=} 0
\end{gathered}
$$

The equation (a) holds due to the fact that LoS component is constant, and equation (b) holds due to the fact that the last term $\tilde{\mathbf{h}}_{r e}$ obeys the Gaussian distribution with mean value of 0 , then it is easy to derive that $t_{2}$ also obeys the Gaussian distribution with mean value of 0 . Similarly, we can have:

$$
\begin{aligned}
& E\left(t_{3}\right)=E\left(t_{4}\right)=E\left(t_{5}\right)=E\left(t_{6}\right) \\
= & E\left(t_{8}\right)=E\left(t_{9}\right)=E\left(t_{11}\right)=E\left(t_{13}\right) \\
= & E\left(t_{14}\right)=E\left(t_{15}\right)=0
\end{aligned}
$$

Then for $t_{7}$, we have

$$
E\left(t_{7}\right)=E\left(\overline{\mathbf{h}}_{r e}^{H} \mathbf{A B}{ }^{H} \tilde{\mathbf{h}}_{r b} \tilde{\mathbf{h}}_{r b}^{H} \mathbf{B} A^{H} \overline{\mathbf{h}}_{r e}\right)
$$

According to the properties of Gaussian distribution, we can derive

$$
E\left(\tilde{\mathbf{h}}_{r b} \tilde{\mathbf{h}}_{r b}^{H}\right)=\left(\begin{array}{cccc}
\sigma_{r b}^{2} & 0 & \cdots & 0 \\
0 & \sigma_{r b}^{2} & & 0 \\
\vdots & & \ddots & \vdots \\
0 & \cdots & & \sigma_{r b}^{2}
\end{array}\right)
$$

Then, we can derive

$$
E\left(t_{7}\right)=\sigma_{r b}^{2} \overline{\mathbf{h}}_{r e}^{H} \mathbf{A} \mathbf{B}^{H} \mathbf{B} A^{H} \overline{\mathbf{h}}_{r e}
$$

Similarly, $t_{10}$ can be expressed as

$$
\begin{gathered}
E\left(t_{10}\right)=E\left(\tilde{\mathbf{h}}_{r e}^{H} \mathbf{A} \mathbf{B}^{H} \overline{\mathbf{h}}_{r b} \overline{\mathbf{h}}_{r b}^{H} \mathbf{B} A^{H} \tilde{\mathbf{h}}_{r e}\right) \\
=\sigma_{r e}^{2} \overline{\mathbf{h}}_{r b}^{H} \mathbf{B} A^{H} \mathbf{A} \mathbf{B}^{H} \overline{\mathbf{h}}_{r b}
\end{gathered}
$$

Similarly, $t_{16}$ can be expressed as

$$
\begin{aligned}
& E\left(t_{16}\right)=E\left(\tilde{\mathbf{h}}_{r e}^{H} A B^{H} \tilde{\mathbf{h}}_{r b} \tilde{\mathbf{h}}_{r b}^{H} B A^{H} \tilde{\mathbf{h}}_{r e}\right) \\
& \quad=\sigma_{r r}^{2} \sigma_{r b}^{2} \operatorname{Tr}\left(A B^{H} B A^{H}\right) \\
& \quad=\sigma_{r e}^{2} \sigma_{r b}^{2} \operatorname{Tr}\left(\Phi_{2} \mathbf{H}_{a r} \mathbf{H}_{a r}^{H} \Phi_{1}^{H} \Phi_{1} \mathbf{H}_{a r} \mathbf{H}_{a r}^{H} \Phi_{2}^{H}\right) \\
& \quad=\sigma_{r e}^{2} \sigma_{r b}^{2} \operatorname{Tr}\left(\mathbf{H}_{a r} \mathbf{H}_{a r}^{H} \mathbf{H}_{a r} \mathbf{H}_{a r}^{H}\right)
\end{aligned}
$$

Further, all the subterm in $p_{16}$ can be summed as follows

$E\left(p_{16}\right)=\overline{\mathbf{h}}_{r e}^{H} \mathbf{A} \mathbf{B}^{H} \overline{\mathbf{h}}_{r b} \overline{\mathbf{h}}_{r b}^{H} \mathbf{B} A^{H} \overline{\mathbf{h}}_{r e}+\sigma_{r b}^{2} \overline{\mathbf{h}}_{r e}^{H} \mathbf{A} \mathbf{B}^{H} \mathbf{B} A^{H} \overline{\mathbf{h}}_{r e}$ $+\sigma_{r e}^{2} \overline{\mathbf{h}}_{r b}^{H} \mathbf{B} A^{H} \mathbf{A} \mathbf{B}^{H} \overline{\mathbf{h}}_{r b}+\sigma_{r e}^{2} \sigma_{r b}^{2} \operatorname{Tr}\left(\mathbf{H}_{a r} \mathbf{H}_{a r}^{H} \mathbf{H}_{a r} \mathbf{H}_{a r}^{H}\right)$

Similarly, for each term in $q_{1}$, we can remove the expected operation and obtain the following result

$$
\begin{aligned}
& E\left(p_{1}\right)=\overline{\mathbf{h}}_{a e}^{H} \overline{\mathbf{h}}_{a b} \overline{\mathbf{h}}_{a b}^{H} \overline{\mathbf{h}}_{a e}+\sigma_{a e}^{2} \overline{\mathbf{h}}_{a b}^{H} \overline{\mathbf{h}}_{a b}+\sigma_{a b}^{2} \overline{\mathbf{h}}_{a e}^{H} \overline{\mathbf{h}}_{a e} \\
& \quad+M_{a} \sigma_{a e}^{2} \sigma_{a b}^{2} \\
& E\left(p_{2}\right)=\overline{\mathbf{h}}_{a e}^{H} \mathbf{B}^{H} \overline{\mathbf{h}}_{r b} \overline{\mathbf{h}}_{r b}^{H} \mathbf{B} \overline{\mathbf{h}}_{a e}+\sigma_{a e}^{2} \overline{\mathbf{h}}_{r b}^{H} \mathbf{B} \overline{\mathbf{h}}_{a b} \\
& E\left(p_{3}\right)=\overline{\mathbf{h}}_{a e}^{H} \overline{\mathbf{h}}_{a b} \overline{\mathbf{h}}_{a b}^{H} \mathbf{A}^{H} \overline{\mathbf{h}}_{r e}+\sigma_{a b}^{2} \overline{\mathbf{h}}_{a e}^{H} \mathbf{A}^{H} \overline{\mathbf{h}}_{r e} \\
& E\left(p_{4}\right)=\overline{\mathbf{h}}_{a e}^{H} \overline{\mathbf{h}}_{a b} \overline{\mathbf{h}}_{r b}^{H} \mathbf{B} \mathbf{A}^{H} \overline{\mathbf{h}}_{r e} \\
& E\left(p_{5}\right)=\overline{\mathbf{h}}_{a e}^{H} \mathbf{B}^{H} \overline{\mathbf{h}}_{r b} \overline{\mathbf{h}}_{a b}^{H} \overline{\mathbf{h}}_{a e}+\sigma_{a e}^{2} \overline{\mathbf{h}}_{a b}^{H} \mathbf{B}^{H} \overline{\mathbf{h}}_{r b} \\
& E\left(p_{6}\right)=\overline{\mathbf{h}}_{a e}^{H} \mathbf{B}^{H} \overline{\mathbf{h}}_{r b} \overline{\mathbf{h}}_{r b}^{H} \mathbf{B} \overline{\mathbf{h}}_{a e}+\sigma_{a e}^{2} \overline{\mathbf{h}}_{r b}^{H} \mathbf{B} \mathbf{B}^{H} \overline{\mathbf{h}}_{r b} \\
& \quad+\sigma_{r b}^{2} \overline{\mathbf{h}}_{a e}^{H} \mathbf{B}^{H} \mathbf{B} \overline{\mathbf{h}}_{a e}+\sigma_{a e}^{2} \sigma_{r b}^{2} \operatorname{Tr}\left(\mathbf{h}_{a r} \mathbf{H}_{a r}^{H}\right) \\
& E\left(p_{7}\right)=\overline{\mathbf{h}}_{a e}^{H} \mathbf{B}^{H} \overline{\mathbf{h}}_{r b} \overline{\mathbf{h}}_{a b}^{H} \mathbf{A}^{H} \overline{\mathbf{h}}_{r e} \\
& E\left(p_{8}\right)=\overline{\mathbf{h}}_{a e}^{H} \mathbf{B}^{H} \overline{\mathbf{h}}_{r b} \overline{\mathbf{h}}_{r b}^{H} \mathbf{B} \mathbf{A}^{H} \overline{\mathbf{h}}_{r e}+\sigma_{r b}^{2} \overline{\mathbf{h}}_{a e}^{H} \mathbf{B}^{H} \mathbf{B} \mathbf{A}^{H} \overline{\mathbf{h}}_{r e} \\
& E\left(p_{9}\right)=\overline{\mathbf{h}}_{r e}^{H} \mathbf{A} \overline{\mathbf{h}}_{a b} \overline{\mathbf{h}}_{a b}^{H} \overline{\mathbf{h}}_{a e}+\sigma_{a b}^{2} \overline{\mathbf{h}}_{r e}^{H} \mathbf{A} \overline{\mathbf{h}}_{a e} \\
& E\left(p_{10}\right)=\overline{\mathbf{h}}_{r e}^{H} A \overline{\mathbf{h}}_{a b} \overline{\mathbf{h}}_{r b}^{H} B \overline{\mathbf{h}}_{a e} \\
& E\left(p_{11}\right)=\overline{\mathbf{h}}_{r e}^{H} \mathbf{A} \overline{\mathbf{h}}_{a b} \overline{\mathbf{h}}_{a b}^{H} \mathbf{A}^{H} \overline{\mathbf{h}}_{r e}+\sigma_{r e}^{2} \mathbf{h}_{a b}^{H} \mathbf{A}^{H} \mathbf{A} \mathbf{h} \\
& \quad+\sigma_{a b}^{2} \overline{\mathbf{h}}_{r e}^{H} \mathbf{A} \mathbf{A}^{H} \overline{\mathbf{h}}_{r e}+\sigma_{a b}^{2} \sigma_{r e}^{2} \operatorname{Tr}\left(\mathbf{H}_{a r} \mathbf{H}_{a r}^{H}\right) \\
& E\left(p_{12}\right)=\overline{\mathbf{h}}_{r e}^{H} A \overline{\mathbf{h}}_{a b} \overline{\mathbf{h}}_{r b}^{H} B A^{H} \overline{\mathbf{h}}_{r e}+\sigma_{r e}^{2} \overline{\mathbf{h}}_{r b}^{H} B A^{H} A \overline{\mathbf{h}}_{a b} \\
& E\left(p_{13}\right)=\overline{\mathbf{h}}_{r e}^{H} \mathbf{A} \mathbf{B}^{H} \overline{\mathbf{h}}_{r b} \overline{\mathbf{h}}_{a b}^{H} \overline{\mathbf{h}}_{a e} \\
& E\left(p_{14}\right)=\overline{\mathbf{h}}_{r e}^{H} \mathbf{A} \mathbf{B}^{H} \overline{\mathbf{h}}_{r b} \overline{\mathbf{h}}_{r b}^{H} B \overline{\mathbf{h}}_{a e}+\sigma_{r b}^{2} \overline{\mathbf{h}}_{r e}^{H} \mathbf{A} \mathbf{B}^{H} \mathbf{B} \overline{\mathbf{h}}_{a e} \\
& E\left(p_{15}\right)=\overline{\mathbf{h}}_{r e}^{H} \mathbf{A} \mathbf{B}^{H} \overline{\mathbf{h}}_{r b} \overline{\mathbf{h}}_{a b}^{H} \mathbf{A}^{H} \overline{\mathbf{h}}_{r e}+\sigma_{r e}^{2} \overline{\mathbf{h}}_{a b}^{H} \mathbf{A}^{H} \mathbf{A} \mathbf{B}^{H} \overline{\mathbf{h}}_{r b}
\end{aligned}
$$


There are many items in (40). It is difficult to merge them directly. We combine some items according to certain association and get $s_{11}, s_{12}, s_{13}, s_{14}, s_{15}$ as follows

$$
\begin{aligned}
& s_{11}=\overline{\mathbf{h}}_{a e}^{H} \overline{\mathbf{h}}_{a b} \overline{\mathbf{h}}_{a b}^{H} \overline{\mathbf{h}}_{a e}+\overline{\mathbf{h}}_{a e}^{H} \mathbf{B}^{H} \overline{\mathbf{h}}_{r b} \overline{\mathbf{h}}_{r b}^{H} \mathbf{B} \overline{\mathbf{h}}_{a e} \\
& +\overline{\mathbf{h}}_{a e}^{H} \overline{\mathbf{h}}_{a b} \overline{\mathbf{h}}_{a b}^{H} \mathbf{A}^{H} \overline{\mathbf{h}}_{r e}+\overline{\mathbf{h}}_{a e}^{H} \overline{\mathbf{h}}_{a b} \overline{\mathbf{h}}_{r b}^{H} \mathbf{B} \mathbf{A}^{H} \overline{\mathbf{h}}_{r e} \\
& +\overline{\mathbf{h}}_{a e}^{H} \mathbf{B}^{H} \overline{\mathbf{h}}_{r b}^{a b} \overline{\mathbf{h}}_{a b}^{H} \overline{\mathbf{h}}_{a e}+\overline{\mathbf{h}}_{a e}^{H} \mathbf{B}^{H} \overline{\mathbf{h}}_{r b} \overline{\mathbf{h}}_{r b}^{H} \mathbf{B} \overline{\mathbf{h}}_{a e} \\
& +\overline{\mathbf{h}}_{a e}^{H} \mathbf{B}^{H} \overline{\mathbf{h}}_{r \underline{\mathbf{h}}}^{r b} \overline{\mathbf{h}}_{a b}^{H b} \mathbf{A}^{H} \overline{\mathbf{h}}_{r e}+\overline{\mathbf{h}}_{a e}^{H} \mathbf{B}^{H} \overline{\mathbf{h}}_{r b}^{r b} \overline{\mathbf{h}}_{r b}^{H} \mathbf{B} \mathbf{A}^{H} \overline{\mathbf{h}}_{r e} \\
& +\overline{\mathbf{h}}_{r e}^{H} \mathbf{A} \overline{\mathbf{h}}_{a b} \overline{\mathbf{h}}_{a b}^{H} \overline{\mathbf{h}}_{a e}+\overline{\mathbf{h}}_{r e}^{H} A \overline{\mathbf{h}}_{a b} \overline{\mathbf{h}}_{r b}^{H} B \overline{\mathbf{h}}_{a e} \\
& +\overline{\mathbf{h}}_{r e}^{H} \mathbf{A} \overline{\mathbf{h}}_{a b} \overline{\mathbf{h}}_{a b}^{H} \mathbf{A}^{H} \overline{\mathbf{h}}_{r e}+\overline{\mathbf{h}}_{r e}^{H} A \overline{\mathbf{h}}_{a b} \overline{\mathbf{h}}_{r b}^{H} B A^{H} \overline{\mathbf{h}}_{r e} \\
& +\overline{\mathbf{h}}_{r e}^{H} \mathbf{A B} \mathbf{B}^{H} \overline{\mathbf{h}}_{r b} \overline{\mathbf{h}}_{a b}^{H} \overline{\mathbf{h}}_{a e}+\overline{\mathbf{h}}_{r e}^{H} \mathbf{A} \mathbf{B}^{H} \overline{\mathbf{h}}_{r b} \overline{\mathbf{h}}_{r b}^{H} \mathbf{B} \overline{\mathbf{h}}_{a e} \\
& +\overline{\mathbf{h}}_{r e}^{H} \mathbf{A} \mathbf{B}^{H} \overline{\mathbf{h}}_{r b} \overline{\mathbf{h}}_{a b}^{H} \mathbf{A}^{H} \overline{\mathbf{h}}_{r e}+\overline{\mathbf{h}}_{r e}^{H} \mathbf{A} \mathbf{B}^{H} \overline{\mathbf{h}}_{r b} \overline{\mathbf{h}}_{r b}^{H} \mathbf{B} A^{H} \overline{\mathbf{h}}_{r e} \\
& =\left|\left(\overline{\mathbf{h}}_{a e}^{H}+\overline{\mathbf{h}}_{r e}^{H} \mathbf{A}\right)\left(\overline{\mathbf{h}}_{a b}+\mathbf{B}^{H} \overline{\mathbf{h}}_{r b}\right)\right|^{2} \\
& =\left|\left(\overline{\mathbf{h}}_{a e}^{H}+\overline{\mathbf{h}}_{r e}^{H} \Phi_{2} \mathbf{H}_{a r}\right)\left(\overline{\mathbf{h}}_{a b}+\mathbf{H}_{a r}^{H} \Phi_{1}^{H} \overline{\mathbf{h}}_{r b}\right)\right|^{2} \\
& s_{12}=\sigma_{a e}^{2} \overline{\mathbf{h}}_{a b}^{H} \overline{\mathbf{h}}_{a b}+\sigma_{a e}^{2} \overline{\mathbf{h}}_{r b}^{H} \mathbf{B} \overline{\mathbf{h}}_{a b} \\
& +\sigma_{a e}^{2} \overline{\mathbf{h}}_{a b}^{H} \mathbf{B}^{H} \overline{\mathbf{h}}_{r b}+\sigma_{a e}^{2} \overline{\mathbf{h}}_{r b}^{H} \mathbf{B} \mathbf{B}^{H} \overline{\mathbf{h}}_{r b} \\
& =\sigma_{a e}^{2}\left|\left(\overline{\mathbf{h}}_{r b}^{H} \mathbf{B}+\overline{\mathbf{h}}_{a b}^{H}\right)\right|^{2} \\
& =\sigma_{a e}^{2}\left|\left(\overline{\mathbf{h}}_{r b}^{H} \Phi_{1} \mathbf{H}_{a r}+\overline{\mathbf{h}}_{a b}^{H}\right)\right|^{2} \\
& s_{13}=\sigma_{a b}^{2} \overline{\mathbf{h}}_{a e}^{H} \overline{\mathbf{h}}_{a e}+\sigma_{a b}^{2} \overline{\mathbf{h}}_{a e}^{H} \mathbf{A}^{H} \overline{\mathbf{h}}_{r e} \\
& +\sigma_{a b}^{2} \overline{\mathbf{h}}_{r e}^{H} \mathbf{A} \overline{\mathbf{h}}_{a e}+\sigma_{a b}^{2} \overline{\mathbf{h}}_{r e}^{H} \mathbf{A} \mathbf{A}^{H} \overline{\mathbf{h}}_{r e} \\
& =\sigma_{a b}^{2}\left|\left(\overline{\mathbf{h}}_{r e}^{H} \mathbf{A}+\overline{\mathbf{h}}_{a e}^{H}\right)\right|^{2} \\
& =\sigma_{a b}^{2}\left|\left(\overline{\mathbf{h}}_{r e}^{H} \Phi_{2} \mathbf{H}_{a r}+\overline{\mathbf{h}}_{a e}^{H}\right)\right|^{2} \\
& s_{14}=\sigma_{r b}^{2} \overline{\mathbf{h}}_{a e}^{H} \mathbf{B}^{H} \mathbf{B} \overline{\mathbf{h}}_{a e}+\sigma_{r b}^{2} \overline{\mathbf{h}}_{a e}^{H} \mathbf{B}^{H} \mathbf{B} \mathbf{A}^{H} \overline{\mathbf{h}}_{r e} \\
& +\sigma_{r b}^{2} \overline{\mathbf{h}}_{a e}^{H} \mathbf{B}^{H} B \overline{\mathbf{h}}_{a e}+\sigma_{r b}^{2} \overline{\mathbf{h}}_{r e}^{H} \mathbf{A} \mathbf{B}^{H} \mathbf{B} \overline{\mathbf{h}}_{a e} \\
& +\sigma_{r b}^{2} \overline{\mathbf{h}}_{r e}^{H} \mathbf{A B}{ }^{H} \mathbf{B} A^{H} \overline{\mathbf{h}}_{r e} \\
& =\sigma_{r b}^{2}\left|\left(\overline{\mathbf{h}}_{r e}^{H} \mathbf{A}+\overline{\mathbf{h}}_{a e}^{H}\right) \mathbf{B}^{H}\right|^{2} \\
& =\sigma_{r b}^{2}\left|\left(\overline{\mathbf{h}}_{r e}^{H} \Phi_{2} \mathbf{H}_{a r}+\overline{\mathbf{h}}_{a e}^{H}\right) \mathbf{H}_{a r}^{H}\right|^{2} \\
& s_{15}=\sigma_{r e}^{2} \mathbf{h}_{a b}^{H} \mathbf{A}^{H} \mathbf{A} \mathbf{h}_{a b}+\sigma_{r e}^{2} \overline{\mathbf{h}}_{r b}^{H} \mathbf{B} \mathbf{A}^{H} \mathbf{A} \overline{\mathbf{h}}_{a b} \\
& +\sigma_{r e}^{2} \overline{\mathbf{h}}_{a b}^{H} \mathbf{A}^{H} \mathbf{A} \mathbf{B}^{H} \overline{\mathbf{h}}_{r b}+\sigma_{r e}^{2} \overline{\mathbf{h}}_{r b}^{H} \mathbf{B} \mathbf{A}^{H} \mathbf{A} \mathbf{B}^{H} \overline{\mathbf{h}}_{r b} \\
& =\sigma_{r e}^{2}\left|\left(\overline{\mathbf{h}}_{r b}^{H} \mathbf{B}+\overline{\mathbf{h}}_{a b}^{H}\right) \mathbf{A}^{H}\right|^{2} \\
& =\sigma_{r e}^{2}\left|\left(\overline{\mathbf{h}}_{r b}^{H} \Phi_{1} \mathbf{H}_{a r}+\overline{\mathbf{h}}_{a b}^{H}\right) \mathbf{H}_{a r}^{H}\right|^{2}
\end{aligned}
$$

Finaly, the expectation of $q_{1}$ can be written as

$$
\begin{aligned}
& E\left(q_{1}\right)=s_{11}+s_{12}+s_{13}+s_{14}+s_{15} \\
& \quad+M_{a} \sigma_{a e}^{2} \sigma_{a b}^{2}+\sigma_{a e}^{2} \sigma_{r b}^{2} \operatorname{Tr}\left(\mathbf{H}_{a r} \mathbf{H}_{a r}^{H}\right) \\
& \quad+\sigma_{a b}^{2} \sigma_{r e}^{2} \operatorname{Tr}\left(\mathbf{H}_{a r} \mathbf{H}_{a r}^{H}\right)+\sigma_{r e}^{2} \sigma_{r b}^{2} \operatorname{Tr}\left(\mathbf{H}_{a r} \mathbf{H}_{a r}^{H} \mathbf{H}_{a r} \mathbf{H}_{a r}^{H}\right) \\
& \quad=\left|\left(\overline{\mathbf{h}}_{a e}^{H}+\overline{\mathbf{h}}_{r e}^{H} \Phi_{2} \mathbf{H}_{a r}\right)\left(\overline{\mathbf{h}}_{a b}+\mathbf{H}_{a r}^{H} \Phi_{1}^{H} \overline{\mathbf{h}}_{r b}\right)\right|^{2} \\
& \quad+\sigma_{a e}^{2}\left|\left(\overline{\mathbf{h}}_{r b}^{H} \Phi_{1} \mathbf{H}_{a r}+\overline{\mathbf{h}}_{a b}^{H}\right)\right|^{2}+\sigma_{a b}^{2}\left|\left(\overline{\mathbf{h}}_{r e}^{H} \Phi_{2} \mathbf{H}_{a r}+\overline{\mathbf{h}}_{a e}^{H}\right)\right|^{2} \\
& \quad+\sigma_{r b}^{2}\left|\left(\overline{\mathbf{h}}_{r e}^{H} \Phi_{2} \mathbf{H}_{a r}+\overline{\mathbf{h}}_{a e}^{H}\right) \mathbf{H}_{a r}^{H}\right|^{2}+\sigma_{r e}^{2} \mid\left(\overline{\mathbf{h}}_{r b}^{H} \Phi_{1} \mathbf{H}_{a r}+\overline{\mathbf{h}}_{a b}^{H}\right) \mathbf{H}_{a r}^{H} \\
& \quad+M_{a} \sigma_{a e}^{2} \sigma_{a b}^{2}+\sigma_{a e}^{2} \sigma_{r b}^{2} \operatorname{Tr}\left(\mathbf{H}_{a r} \mathbf{H}_{a r}^{H}\right)+\sigma_{a b}^{2} \sigma_{r e}^{2} \operatorname{Tr}\left(\mathbf{H}_{a r} \mathbf{H}_{a r}^{H}\right) \\
& \quad+\sigma_{r e}^{2} \sigma_{r b}^{2} \operatorname{Tr}\left(\mathbf{H}_{a r} \mathbf{H}_{a r}^{H} \mathbf{H}_{a r} \mathbf{H}_{a r}^{H}\right)
\end{aligned}
$$

In the same way, the expectation of $q_{2}$ can be derived as

$$
\begin{aligned}
& E\left(q_{2}\right)=E\left(\left\|\mathbf{H}_{a r}^{H} \Phi_{1}^{H}\left(\overline{\mathbf{h}}_{r b}+\tilde{\mathbf{h}}_{r b}\right)\right\|_{2}^{2}\right) \\
& \quad=\left|\left(\overline{\mathbf{h}}_{r b}^{H} \mathbf{B}+\overline{\mathbf{h}}_{a b}^{H}\right)\right|^{2}+\sigma_{r b}^{2} \operatorname{Tr}\left(\mathbf{H}_{a r} \mathbf{H}_{a r}^{H}\right)+M_{a} \sigma_{a b}^{2}
\end{aligned}
$$

Therefore, the expectation expression of the denominator $\mathrm{E}\left(g_{1}\left(\Phi_{1}, \Phi_{2}\right)\right)$ can be obtained.
Since the form of the numerator is similar to that of denominator in (11), the expectation expression of the denominator can be derived as

$$
\begin{aligned}
& f_{2}\left(\Phi_{1}, \Phi_{2}\right) \\
& =\mathrm{E}\left(f_{1}\left(\Phi_{1}, \Phi_{2}\right)\right) \\
& =P_{t}\left|\left(\overline{\mathbf{h}}_{a b}^{H}+\overline{\mathbf{h}}_{r b}^{H} \Phi_{2} \mathbf{H}_{a r}\right)\left(\overline{\mathbf{h}}_{a b}+\mathbf{H}_{a r}^{H} \Phi_{1}^{H} \overline{\mathbf{h}}_{r b}\right)\right|^{2} \\
& +P_{t} \sigma_{a b}^{2}\left|\left(\overline{\mathbf{h}}_{r b}^{H} \Phi_{1} \mathbf{H}_{a r}+\overline{\mathbf{h}}_{a b}^{H}\right)\right|^{2}+P_{t} \sigma_{a b}^{2}\left|\left(\overline{\mathbf{h}}_{r b}^{H} \Phi_{2} \mathbf{H}_{a r}+\overline{\mathbf{h}}_{a b}^{H}\right)\right|^{2} \\
& +P_{t} \sigma_{r b}^{2}\left|\left(\overline{\mathbf{h}}_{r b}^{H} \Phi_{2} \mathbf{H}_{a r}+\overline{\mathbf{h}}_{a b}^{H}\right) \mathbf{H}_{a r}^{H}\right|^{2}+\sigma^{2}\left|\left(\overline{\mathbf{h}}_{r b}^{H} \Phi_{1} \mathbf{H}_{a r}+\overline{\mathbf{h}}_{a b}^{H}\right)\right|^{2} \\
& +P_{t} \sigma_{r b}^{2}\left|\left(\overline{\mathbf{h}}_{r b}^{H} \Phi_{1} \mathbf{H}_{a r}+\overline{\mathbf{h}}_{a b}^{H}\right) \mathbf{H}_{a r}^{H}\right|^{2}+\sigma^{2} \sigma_{r b}^{2} \operatorname{Tr}\left(\mathbf{H}_{a r} \mathbf{H}_{a r}^{H}\right) \\
& +M_{a} \sigma^{2} \sigma_{a b}^{2}+P_{t} M_{a} \sigma_{a b}^{4}+2 P_{t} \sigma_{a b}^{2} \sigma_{r b}^{2} \operatorname{Tr}\left(\mathbf{H}_{a r} \mathbf{H}_{a r}^{H}\right) \\
& +P_{t} \sigma_{r b}^{4} \operatorname{Tr}\left(\mathbf{H}_{a r} \mathbf{H}_{a r}^{H} \mathbf{H}_{a r} \mathbf{H}_{a r}^{H}\right)
\end{aligned}
$$

Thus, the proof is completed.

\section{REFERENCES}

[1] J. M. Hamamreh, H. M. Furqan, and H. Arslan, "Classifications and applications of physical layer security techniques for confidentiality: A comprehensive survey," IEEE Communications Surveys \& Tutorials, vol. PP, pp. 1-1, 2018.

[2] X. Zhou, B. Maham, and A. Hjorungnes, "Pilot contamination for active eavesdropping," IEEE Transactions on Wireless Communications, vol. 11, no. 3, pp. 903-907, 2012.

[3] K.-W. Huang, H.-M. Wang, Y. Wu, and R. Schober, "Pilot spoofing attack by multiple eavesdroppers," IEEE Transactions on Wireless Communications, vol. 17, no. 10, pp. 6433-6447, 2018.

[4] Q. Xiong, Y.-C. Liang, K. H. Li, and Y. Gong, "An energy-ratiobased approach for detecting pilot spoofing attack in multiple-antenna systems," IEEE Transactions on Information Forensics and Security, vol. 10, no. 5, pp. 932-940, 2015.

[5] X. Tian, M. Li, and Q. Liu, "Random-training-assisted pilot spoofing detection and security enhancement," IEEE Access, vol. 5, pp. 27384 27399,2017

[6] J. Zhao and Y. Liu, "A survey of intelligent reflecting surfaces (irss): Towards $6 \mathrm{~g}$ wireless communication networks," arXiv preprint arXiv:1907.04789, 2019.

[7] Q. Wu, S. Zhang, B. Zheng, C. You, and R. Zhang, "Intelligent reflecting surface aided wireless communications: A tutorial," arXiv preprint arXiv:2007.02759, 2020.

[8] H. Guo, Y.-C. Liang, J. Chen, and E. G. Larsson, "Weighted sumrate maximization for intelligent reflecting surface enhanced wireless networks," in 2019 IEEE Global Communications Conference (GLOBECOM). IEEE, 2019, pp. 1-6.

[9] M. Cui, G. Zhang, and R. Zhang, "Secure wireless communication via intelligent reflecting surface," IEEE Wireless Communications Letters, vol. 8, no. 5, pp. 1410-1414, 2019.

[10] L. Dong and H. M. Wang, "Secure mimo transmission via intelligent reflecting surface," IEEE Wireless Communications Letters, vol. 9, no. 6 , pp. 787-790, 2020.

[11] C. Huang, A. Zappone, G. C. Alexandropoulos, M. Debbah, and C. Yuen, "Reconfigurable intelligent surfaces for energy efficiency in wireless communication," IEEE Transactions on Wireless Communications, vol. 18, no. 8, pp. 4157-4170, 2019.

[12] B. Lyu, D. T. Hoang, S. Gong, D. Niyato, and D. I. Kim, "Irs-based wireless jamming attacks: When jammers can attack without power," IEEE Wireless Communications Letters, vol. 9, no. 10, pp. 1663-1667, 2020.

[13] A. Taha, M. Alrabeiah, and A. Alkhateeb, "Enabling large intelligent surfaces with compressive sensing and deep learning," arXiv preprint arXiv:1904.10136, 2019.

[14] A. Taha, Y. Zhang, F. B. Mismar, and A. Alkhateeb, "Deep reinforcement learning for intelligent reflecting surfaces: Towards standalone operation," arXiv preprint arXiv:2002.11101, 2020.

[15] J. Moon, S. H. Lee, H. Lee, and I. Lee, "Proactive eavesdropping with jamming and eavesdropping mode selection," IEEE Transactions on Wireless Communications, vol. 18, no. 7, pp. 3726-3738, 2019.

[16] C. Zhong, X. Jiang, F. Qu, and Z. Zhang, "Multi-antenna wireless legitimate surveillance systems: Design and performance analysis," IEEE Transactions on Wireless Communications, vol. PP, no. 7, pp. 1-1, 2017. 
[17] Q. Zhang, S. Jin, K.-K. Wong, H. Zhu, and M. Matthaiou, "Power scaling of uplink massive mimo systems with arbitrary-rank channel means," IEEE Journal of Selected Topics in Signal Processing, vol. 8, no. 5, pp. 966-981, 2014

[18] X. Guan, Q. Wu, and R. Zhang, "Intelligent reflecting surface assisted secrecy communication: Is artificial noise helpful or not?" IEEE Wireless Communications Letters, pp. 1-1, 2020. 